\title{
Flip-Connectivity of Triangulations of the Product of a Tetrahedron and Simplex
}

\author{
Gaku Liu ${ }^{1}$ \\ Received: 2 April 2016 / Revised: 6 June 2019 / Accepted: 13 November 2019 / \\ Published online: 1 December 2019 \\ (c) The Author(s) 2019
}

\begin{abstract}
A flip is a minimal move between two triangulations of a polytope. The set of triangulations of a polytope was shown by Santos to not always be connected by flips, and it is an interesting problem to find large classes of polytopes for which it is. One such class which has received considerable attention is the product of two simplices. Santos proved that the set of triangulations of a product of two simplices is connected by flips when one of the simplices is a triangle. However, the author showed that it is not connected when one of the simplices is four-dimensional and the other has very large dimension. In this paper we show that it is connected when one of the simplices is a tetrahedron, thereby extending Santos's result as far as possible.
\end{abstract}

Keywords Triangulations · Product of simplices · Flip graph

Mathematics Subject Classification 52B20 · 52B11

\section{Introduction}

The triangulations of the product of two simplices are a well-studied and ubiquitous set of objects. They have connections to algebraic geometry and commutative algebra and are an important tool to understanding triangulations of products of other polytopes. See [4, Chap. 6.2] for an overview. They have also been extensively studied for their own sake and have been characterized as generic tropical hyperplane arrangements, fine mixed subdivisions, and certain tropical oriented matroids [3,5,14].

An interesting question that had received considerable attention is whether or not the set of triangulations of the product of two simplices is flip-connected. A flip can be thought of as a minimal move between two triangulations of a fixed polytope, and were originally defined by Gel'fand et al. in their study of discriminants and resultants

\section{Editor in Charge: Kenneth Clarkson}

Gaku Liu

gakuliu@mis.mpg.de

1 Max-Planck-Institut fur Mathematik in den Naturwissenschaften, Leipzig, Germany 
for sparse polynomials [6]. The set of triangulations of a polytope is flip-connected if any two triangulations can be connected by a series of flips. It has long been known that every two-dimensional polygon has a flip-connected set of triangulations. On the other hand, there are examples by Santos in higher dimensions of polytopes with non-flip-connected triangulations [12]. However, there is very little known about flipconnectivity even in specific cases or in low dimension; for example, it is unknown whether all three-dimensional polytopes have flip-connected sets of triangulations.

The question of flip-connectivity for triangulations of the product of two simplices has received particular attention due to these objects' relation with tropical hyperplane arrangements and tropical pseudo-hyperplane arrangements, where flips have a natural interpretation. See $[3,5,7,11]$. In 2005, Santos [14] proved that the triangulations of $\Delta^{2} \times \Delta^{n}$ are flip-connected for all $n$. Recently, however, the author showed that the triangulations of $\Delta^{4} \times \Delta^{n}$ are not flip-connected when $n \approx 4 \cdot 10^{4}$ [9]. The present paper aims to fill in the "gap" by showing the following.

Theorem 1.1 The set of triangulations of $\Delta^{3} \times \Delta^{n}$ is flip-connected for all $n$.

A corollary of this result comes from toric geometry, where each lattice polytope defines a toric ideal and an associated toric Hilbert scheme; see [15, Chap. 10] or [10]. Our result implies the following.

Corollary 1.2 The toric Hilbert scheme of the determinantal ideal of $2 \times 2$ minors of a $4 \times n$ matrix is connected.

Santos's proof for $\Delta^{2} \times \Delta^{n}$ is based on the "Cayley trick", which allows one to visualize triangulations of $\Delta^{m} \times \Delta^{n}$ as tilings of an $m$-dimensional simplex. This leads to an elegant argument based on two-dimensional tilings. For $\Delta^{3} \times \Delta^{n}$ the same trick can be used to convert the problem to one on three-dimensional tilings, and we will follow an overall strategy that is a generalization of the one used by Santos. However, there are many complications that arise when going from two to three dimensions. One is that some triangulations of $\Delta^{3} \times \Delta^{n}$ are "flip-deficient": roughly speaking, they have fewer flips than one would expect given the number of vertices and dimension [4]. This requires us to use a delicate process to even find a flip on certain triangulations.

Our proof gives an algorithm for applying flips to a triangulation until it reaches a specific fixed triangulation. Each iteration of the algorithm consists of two steps:

1. Locate a special circuit of $\Delta^{3} \times \Delta^{n}$, and consider the set $\mathscr{S}$ of all simplices of the triangulation which contain the negative elements of this circuit.

2. Apply a series of flips such that the only simplices affected are in $\mathscr{S}$, until eventually the special circuit itself can be flipped.

Step 1, while taking up a relatively small portion of this paper, is the key idea of the proof. Choosing the circuit correctly heavily restricts the types of simplices that can appear in $\mathscr{S}$ and allows us to find flips. We will find our special circuit using a quasiorder (Sect. 4.1.3) defined on all the simplices of the triangulation.

We will also develop a large amount of general machinery for working with triangulations of products of simplices that may be of independent interest. For example, the ideas in the paper may help in tackling the Spread Out Simplices Conjecture of Ardila and Billey [1]. 
The paper is organized as follows. Section 2 gives an overview of triangulations and flips in general and defines some other important concepts, in particular contraction. Section 3 discusses triangulations of the product of two simplices. Section 4 develops the machinery we will use in the main proof. Section 5 is the main proof.

\section{Triangulations of General Sets}

Our definitions and notation regarding triangulations follow the book of de Loera et al. [4], and we refer to this text for a more thorough treatment.

\subsection{Triangulations and Flips}

Throughout this section, let $A$ be a finite set of points in $\mathbb{R}^{d}$. In this paper, a simplex is an affinely independent set of points. Let $\operatorname{conv}(S)$ denote the convex hull of a point set $S$. Recall that a triangulation of $A$ is a collection $\mathscr{T}$ of simplices which are subsets of $A$ such that

1. Any subset of a simplex in $\mathscr{T}$ is in $\mathscr{T}$.

2. Any two simplices $\sigma_{1}, \sigma_{2} \in \mathscr{T}$ intersect properly; that is, $\operatorname{conv}\left(\sigma_{1}\right) \cap \operatorname{conv}\left(\sigma_{2}\right)=$ $\operatorname{conv}\left(\sigma_{1} \cap \sigma_{2}\right)$

3. $\bigcup_{\sigma \in \mathscr{T}} \operatorname{conv}(\sigma)=\operatorname{conv}(A)$.

Given a triangulation $\mathscr{T}$, let $\mathscr{T}^{*}$ denote the subcollection of maximal simplices of $\mathscr{T}$. Note that $\mathscr{T}$ is determined by $\mathscr{T}^{*}$.

Recall that a circuit is a minimal affinely dependent subset of $\mathbb{R}^{d}$, and that each circuit $X$ has a unique partition $X=X^{+} \sqcup X^{-}$such that relint $\operatorname{conv}\left(X^{+}\right) \cap$ relint conv $\left(X^{-}\right) \neq \varnothing$. We will write this as $X=\left\{X^{+}, X^{-}\right\}$. Any circuit $X=$ $\left\{X^{+}, X^{-}\right\}$has exactly two triangulations, which we denote by

$$
\mathscr{T}_{X}^{+}:=\left\{\sigma \subseteq X: \sigma \nsupseteq X^{+}\right\}, \quad \mathscr{T}_{X}^{-}:=\left\{\sigma \subseteq X: \sigma \nsupseteq X^{-}\right\} .
$$

Given a triangulation $\mathscr{T}$ and a simplex $\sigma \in \mathscr{T}$, we define the link of $\sigma$ in $\mathscr{T}$ as

$$
\operatorname{link}_{\mathscr{T}}(\sigma):=\{\rho \in \mathscr{T}: \rho \cap \sigma=\emptyset, \rho \cup \sigma \in \mathscr{T}\}
$$

We are now ready to state the definition of a flip, in the form of a proposition.

Proposition 2.1 ([13]) Let $\mathscr{T}$ be a triangulation of $A$. Suppose there is a circuit $X=$ $\left\{X^{+}, X^{-}\right\}$contained in A such that

1. $\mathscr{T}_{X}^{+} \subseteq \mathscr{T}$.

2. All maximal simplices of $\mathscr{T}_{X}^{+}$have the same link $\mathscr{L}$ in $\mathscr{T}$.

Then the collection

$$
\mathscr{T}^{\prime}:=\mathscr{T} \backslash\left\{\rho \cup \sigma: \rho \in \mathscr{L}, \sigma \in \mathscr{T}_{X}^{+}\right\} \cup\left\{\rho \cup \sigma: \rho \in \mathscr{L}, \sigma \in \mathscr{T}_{X}^{-}\right\}
$$

is a triangulation of $A$. We say that $\mathscr{T}$ has a flip supported on $\left(X^{+}, X^{-}\right)$, and that $\mathscr{T}^{\prime}$ is the result of applying this flip to $\mathscr{T}$. 
If two triangulations of $A$ can be connected by a series of flips, then we say that these triangulations are flip-connected. If every pair of triangulations of $A$ is flip-connected, then the set of triangulations of $A$ is flip-connected.

\subsection{Local Triangulations and Contractions}

For technical reasons, we sometimes need to deal with "partial" triangulations which do not form convex sets. We make an easy generalization of triangulations to deal with this. Let $A \subseteq \mathbb{R}^{d}$ be a point configuration, and let $\xi$ be a simplex of $A$.

Definition 2.2 For $\xi$ nonempty, a local triangulation of $A$ at $\xi$ is a collection $\mathscr{T}$ of simplices contained in $A$ such that

1. All simplices in $\mathscr{T}$ contain $\xi$.

2. If $\sigma \subseteq \mathscr{T}$ and $\xi \subseteq \sigma^{\prime} \subseteq \sigma$, then $\sigma^{\prime} \subseteq \mathscr{T}$.

3. Any two simplices in $\mathscr{T}$ intersect properly.

4. There is an open set $U \subset \mathbb{R}^{d}$ with $U \cap \xi \neq \emptyset$ such that

$$
\left(\bigcup_{\sigma \in \mathscr{T}} \operatorname{conv}(\sigma)\right) \cap U=\operatorname{conv}(A) \cap U
$$

If $\xi=\emptyset$, then we define a local triangulation of $A$ at $\xi$ to be a triangulation of $A$.

Let $\mathscr{T}^{*}$ denote the set of maximal simplices of a local triangulation $\mathscr{T}$. If $\mathscr{T}$ is a local triangulation of $A$ at (possibly empty) $\zeta$ and $\xi \subseteq A$ is a simplex, let $\mathscr{T}(\xi)$ be the subcollection of simplices of $\mathscr{T}$ that contain $\xi$. If $\xi \cup \zeta \in \mathscr{T}$, then $\mathscr{T}(\xi)$ is a local triangulation of $A$ at $\xi \cup \zeta$.

Suppose $\xi \subseteq A$ is a nonempty simplex, and let $S$ be the intersection of $A$ with the affine span of $\xi$. Later, we will consider the contraction of $A$ with respect to $S$, denoted $A$ / S. Since the definition is somewhat technical, we defer to [4, Sect. 4.2.4] for the exposition. The only fact we will need for the paper is the following:

Proposition 2.3 There is a bijection from the set of local triangulations of A at $\xi$ to the set of triangulations of $A / S$.

\section{The Product of Two Simplices}

\subsection{The Oriented Matroid of $\Delta^{m-1} \times \Delta^{n-1}$}

Let $A=\Delta^{m-1} \times \Delta^{n-1}$, the product of two simplices of dimensions $m-1$ and $n-1$. Following the conventions of the previous section, we will understand $\Delta^{m-1} \times \Delta^{n-1}$ to mean the set of vertices of $\Delta^{m-1} \times \Delta^{n-1}$ rather than the polytope itself. The vertices of $\Delta^{m-1} \times \Delta^{n-1}$ are given by

$$
\left\{e_{i} \times f_{j}: i \in[m], j \in[n]\right\}
$$


where $[l]:=\{1,2, \ldots, l\}$ and $\Delta^{m-1}:=\left\{e_{1}, \ldots, e_{m}\right\}, \Delta^{n-1}:=\left\{f_{1}, \ldots, f_{n}\right\}$. Consider a bipartite directed graph $G_{m, n}$ with vertex set $\Delta^{m-1} \cup \Delta^{n-1}$ and directed edges $e_{i} f_{j}$ for each $i, j$. We have bijection $e_{i} \times f_{j} \mapsto e_{i} f_{j}$ from $\Delta^{m-1} \times \Delta^{n-1}$ to edges of $G_{m, n}$. In this map, the circuits of $\Delta^{m-1} \times \Delta^{n-1}$ map bijectively to undirected cycles of $G_{m, n}$. If we traverse a cycle $C$ of $G_{m, n}$, then we travel along some edges in the forward direction and the other edges in the backward direction; this gives a partition $C^{+} \cup C^{-}$of the edges of $C$. This partition is the same as the partition $X=\left\{X^{+}, X^{-}\right\}$ of the circuit $X \subseteq \Delta^{m-1} \times \Delta^{n-1}$ corresponding to $C$.

All of this can be summarized by saying that the map $e_{i} \times f_{j} \mapsto e_{i} f_{j}$ gives an isomorphism of oriented matroids from the oriented matroid of affine dependencies of $\Delta^{m-1} \times \Delta^{n-1}$ to the oriented matroid of $G_{m, n}$.

Through the above map, each simplex $\sigma \subseteq \Delta^{m-1} \times \Delta^{n-1}$ maps to a set of edges $E(\sigma)$ of $G_{m, n}$ with no cycles. Let $T(\sigma)$ denote the graph with vertex set $\Delta^{m-1} \cup \Delta^{n-1}$ and edge set $E(\sigma)$. If $\sigma$ is maximal, then $T(\sigma)$ is a spanning tree of $G_{m, n}$.

Let $\sigma \subseteq \Delta^{m-1} \times \Delta^{n-1}$ be a simplex. For each vertex $v$ of $G_{m, n}$, define $N_{\sigma}(v)$ to be the undirected neighborhood of $v$ in $T(\sigma)$. Suppose that $j_{1}, \ldots, j_{k}$ are all the indices $j \in[n]$ for which $\left|N_{\sigma}\left(f_{j}\right)\right|>1$. We define the shape ${ }^{1}$ of $\sigma$ to be the set

$$
\operatorname{shape}(\sigma):=\left\{N_{\sigma}\left(f_{j_{1}}\right), \ldots, N_{\sigma}\left(f_{j_{k}}\right)\right\}
$$

We call a simplex with shape $\left\{\Delta^{m-1}\right\}$ an unmixed simplex. If $\sigma$ is unmixed, then $\sigma$ is maximal, and there is a unique $f_{j} \in \Delta^{n-1}$ such that $N_{\sigma}\left(f_{j}\right)=\Delta^{m-1}$. In this case, we "label" $\sigma$ with $f_{j}$. If $\mathscr{T}$ is a triangulation, this labeling gives a bijection between $\Delta^{n-1}$ and the set of unmixed simplices of $\mathscr{T}$.

\subsection{Triangulations of $\Delta^{1} \times \Delta^{n-1}$}

The case of $\Delta^{1} \times \Delta^{n-1}$ will be an important starting point for our argument. Note that every simplex of $\Delta^{1} \times \Delta^{n-1}$ is unmixed and thus has a label in $\Delta^{n-1}$. The triangulations and local triangulations of $\Delta^{1} \times \Delta^{n-1}$ are well known and easy to describe explicitly; see [4, Sect. 6.2]. We rephrase their description as follows:

Proposition 3.1 Let $\mathscr{T}$ be a local triangulation of $\Delta^{1} \times \Delta^{n-1}$ at $\xi \subseteq\left\{e_{1} \times f_{1}, e_{2} \times f_{2}\right\}$. Then there exists a unique ordering $\tau_{1}, \ldots, \tau_{N}$ of $\mathscr{T}^{*}$ such that if $f_{j_{r}}$ is the label of $\tau_{r}$, then:

1. For each $r=1, \ldots, N-1, \tau_{r}$ is adjacent to $\tau_{r+1}$, and these are the only pairs of adjacent maximal simplices.

2. The $f_{j_{r}}$ are distinct, and

$$
\tau_{r+1}=\tau_{r} \backslash\left\{e_{1} \times f_{j_{r}}\right\} \cup\left\{e_{2} \times f_{j_{r+1}}\right\}
$$

\section{Furthermore,}

3. If $e_{1} \times f_{1} \in \xi$, then $f_{j_{N}}=f_{1}$; otherwise, $N_{\tau_{N}}\left(e_{2}\right)=\Delta^{n-1}$.

\footnotetext{
1 The term "shape" refers to the shape of the corresponding fine mixed cell after performing the Cayley trick. See [14].
} 
4. If $e_{2} \times f_{2} \in \xi$, then $f_{j_{1}}=f_{2} ;$ otherwise, $N_{\tau_{1}}\left(e_{1}\right)=\Delta^{n-1}$.

Let $\tau_{1}, \ldots, \tau_{N}$ be the ordering of the maximal simplices of $\mathscr{T}$ given by Proposition 3.1. We formalize this order as $<\mathscr{T}$, so that

$$
\tau_{1}<\mathscr{T} \tau_{2}<\mathscr{T}<\cdots<\mathscr{T} \tau_{N}
$$

Let $f_{j_{1}}, \ldots, f_{j_{N}}$ be as in the proposition. We have a total order $<\mathscr{T}$ on the $\left\{f_{j_{r}}\right\}$ given by

$$
f_{j_{1}}<\mathscr{T} f_{j_{2}}<\mathscr{T} \cdots<\mathscr{T} f_{j_{N}}
$$

We can interpret these orders as follows: If $j \in\left\{j_{1}, \ldots, j_{N}\right\}$, then any simplex of $\mathscr{T}$ with a label greater than or equal to $f_{j}$ contains $e_{2} \times f_{j}$, and any maximal simplex with a label less than or equal to $f_{j}$ contains $e_{1} \times f_{j}$.

We can use this idea to extend $<\mathscr{T}$ to a quasiorder on $\Delta^{n-1}$ as follows. Let $f_{j_{1}^{\prime}}, \ldots, f_{j_{N^{\prime}}^{\prime}}$ be the elements of $N_{\tau_{1}}\left(e_{2}\right) \backslash\left\{f_{j_{1}}\right\}$, and let $f_{j_{1}^{\prime \prime}}, \ldots, f_{j_{N^{\prime \prime}}^{\prime \prime}}$ be the elements of $N_{\tau_{N}}\left(e_{1}\right) \backslash\left\{f_{j_{N}}\right\}$. We define the quasiorder $\leq \mathscr{T}$ on $\Delta^{n-1}$ by

$$
f_{j_{1}^{\prime}}=\mathscr{T} \cdots=\mathscr{T} f_{j_{N^{\prime}}^{\prime}}<\mathscr{T} f_{j_{1}}<\mathscr{T} \cdots<\mathscr{T} f_{j_{N}}<\mathscr{T} f_{j_{1}^{\prime \prime}}=\mathscr{T} \cdots=\mathscr{T} f_{j_{N^{\prime \prime}}^{\prime \prime}} \cdot(3
$$

If $\mathscr{T}$ is a triangulation, then $\leq \mathscr{T}$ is a total order on $\Delta^{n-1}$.

We have the following easy way to determine the relative order of two elements.

Proposition 3.2 Let $f_{j}, f_{j^{\prime}} \in \Delta^{m-1}$ be distinct. Then $f_{j}<\mathscr{T} f_{j^{\prime}}$ if and only if

$$
\left\{e_{2} \times f_{j}, e_{1} \times f_{j^{\prime}}\right\} \subseteq \sigma \in \mathscr{T}
$$

for some $\sigma$.

Proof Suppose $f_{j}<\mathscr{T} f_{j^{\prime}}$. If there is a maximal simplex of $\mathscr{T}$ with label $f_{j}$, let $\tau$ be that simplex. Otherwise, since $f_{j}<\mathscr{T} f_{j^{\prime}}$, we must have $f_{j} \in N_{\tau_{1}}\left(e_{2}\right) \backslash\left\{f_{j_{1}}\right\}$; in this case, let $\tau=\tau_{1}$. Similarly, let $\tau^{\prime}$ be the maximal simplex of $\mathscr{T}$ with label $f_{j^{\prime}}$ if such a simplex exists, and let $\tau^{\prime}=\tau_{N}$ otherwise. In any case, we have $e_{2} \times f_{j} \in \tau$, $e_{1} \times f_{j^{\prime}} \in \tau^{\prime}$, and $\tau \leq \mathscr{T} \tau^{\prime}$. By Proposition 3.1, we have $N_{\tau}\left(e_{1}\right) \supseteq N_{\tau^{\prime}}\left(e_{1}\right)$. Hence $\left\{e_{2} \times f_{j}, e_{1} \times f_{j^{\prime}}\right\} \subseteq \tau \in \mathscr{T}$, as desired.

Conversely, suppose that $\left\{e_{2} \times f_{j}, e_{1} \times f_{j^{\prime}}\right\} \subseteq \sigma \in \mathscr{T}$. Let $\tau_{0}$ be a maximal simplex of $\mathscr{T}$ containing $\sigma$. Let $\tau$ be the smallest simplex in the order (3.1) containing $e_{2} \times f_{j}$, and let $\tau^{\prime}$ be the largest simplex in this order containing $e_{1} \times f_{j^{\prime}}$. By Proposition 3.1, either $\tau$ has label $f_{j}$ or $f_{j} \in N_{\tau_{1}}\left(e_{2}\right) \backslash\left\{f_{j_{1}}\right\}$. Similarly, either $\tau^{\prime}$ has label $f_{j^{\prime}}$ or $f_{j^{\prime}} \in N_{\tau_{N}}\left(e_{1}\right) \backslash\left\{f_{j_{N}}\right\}$. In any case, since $\tau \leq \mathscr{T} \tau_{0} \leq \mathscr{T} \tau^{\prime}$ and $f_{j}$ and $f_{j^{\prime}}$ are distinct, we have $f_{j}<\mathscr{T} f_{j^{\prime}}$.

\subsection{Restriction and Contraction}

In this section we look at restrictions and contractions of triangulations of $\Delta^{m-1} \times$ $\Delta^{n-1}$. 
The faces of $\Delta^{m-1} \times \Delta^{n-1}$ are the sets $I \times J$ where $I \subseteq \Delta^{m-1}$ and $J \subseteq \Delta^{n-1}$. Given a triangulation $\mathscr{T}$ of $\Delta^{m-1} \times \Delta^{n-1}$, the restriction of $\mathscr{T}$ to $I \times J$ is

$$
\mathscr{T}[I \times J]:=\{\sigma \in \mathscr{T}: \sigma \subseteq I \times J\}
$$

We now consider contraction. Let $\xi \subseteq \Delta^{m-1} \times \Delta^{n-1}$ be a simplex. Let $I=$ $\left(I_{1}, \ldots, I_{S}\right)$ be a partition of $\Delta^{m-1}$ such that each $I_{r}$ is the intersection of $\Delta^{m-1}$ with a connected component of $T(\xi)$. Let $J=\left(J_{1}, \ldots, J_{t}\right)$ be a partition of $\Delta^{n-1}$ such that each $J_{r}$ is the intersection of $\Delta^{n-1}$ with a connected component of $T(\xi)$. We say that these partitions are associated to $\xi$.

Let $C_{1}, \ldots, C_{k}$ be the connected components of $T(\xi)$ containing an edge, and let $p_{1}, \ldots, p_{k}$ and $q_{1}, \ldots, q_{k}$ be the indices such that for each $r=1, \ldots, k, I_{p_{r}} \cup J_{q_{r}}$ is the vertex set of $C_{r}$. Then the intersection of $\Delta^{m-1} \times \Delta^{n-1}$ with the affine span of $\xi$ is

$$
S:=\left(I_{p_{1}} \times J_{q_{1}}\right) \cup \cdots \cup\left(I_{p_{k}} \times J_{q_{k}}\right) .
$$

We now compute the contraction $\Delta^{m-1} \times \Delta^{n-1} / S$. Let $V$ be a real vector space with basis $\Delta^{m-1} \cup \Delta^{n-1}$, and embed $\Delta^{m-1} \times \Delta^{n-1} \subset V$ as $e_{i} \times f_{j} \mapsto e_{i}-f_{j}$. Let $V^{\prime}$ be a real vector space which has the set of connected components of $T(\xi)$ as a basis. (That is, $V^{\prime}$ consists of formal linear combinations of the connected components of $T(\xi)$.) Let $\pi: V \rightarrow V^{\prime}$ be the linear map which takes $v \in \Delta^{m-1} \cup \Delta^{n-1}$ to the connected component of $T(\xi)$ that contains $v$. Then the kernel of $\pi$ is the linear span of $S$. Hence, we have

$$
\Delta^{m-1} \times \Delta^{n-1} / S=\left\{\pi\left(e_{i} \times f_{j}\right): e_{i} \times f_{j} \notin S\right\}
$$

Moreover, if $C_{r}$ consists of a single edge for all $r$, then $\pi$ is one-to-one on $\Delta^{m-1} \times$ $\Delta^{n-1} \backslash S$.

Recall from Proposition 2.3 that local triangulations at $\xi$ are equivalent to triangulations of $\Delta^{m-1} \times \Delta^{n-1} / S$. To better understand such triangulations, let $I$ and $J$ be as above, and construct simplices

$$
\Delta_{I}:=\left\{\bar{e}_{1}, \ldots, \bar{e}_{s}\right\}, \quad \Delta_{J}:=\left\{\bar{f}_{1}, \ldots, \bar{f}_{t}\right\}
$$

By the above computation, the simplification ${ }^{2}$ of $\Delta^{m-1} \times \Delta^{n-1} / S$ is isomorphic to

$$
\Delta_{I} \times \Delta_{J} /\left\{\bar{e}_{p_{1}} \times \bar{f}_{q_{1}}, \ldots, \bar{e}_{p_{k}} \times \bar{f}_{q_{k}}\right\}
$$

It follows that every triangulation of $\Delta^{m-1} \times \Delta^{n-1} / S$ gives a triangulation of $\Delta_{I} \times$ $\Delta_{J} /\left\{\bar{e}_{p_{1}} \times \bar{f}_{q_{1}}, \ldots, \bar{e}_{p_{k}} \times \bar{f}_{q_{k}}\right\}$. Hence, every local triangulation of $\Delta^{m-1} \times \Delta^{n-1}$ at $\xi$ gives a local triangulation of $\Delta_{I} \times \Delta_{J}$ at $\left\{\bar{e}_{p_{1}} \times \bar{f}_{q_{1}}, \ldots, \bar{e}_{p_{k}} \times \bar{f}_{q_{k}}\right\}$.

\footnotetext{
2 As explained in [4], the contraction operation gives a multiset; the simplification is the underlying set.
} 
We now describe this map more explicitly. Let $\phi_{I}: \Delta^{m-1} \rightarrow \Delta_{I}$ and $\phi_{J}: \Delta^{m-1} \rightarrow$ $\Delta_{J}$ be the maps

$$
\phi_{I}\left(e_{i}\right)=\bar{e}_{r} \quad \text { if } e_{i} \in I_{r}, \quad \phi_{J}\left(f_{j}\right)=\bar{f}_{r} \quad \text { if } f_{j} \in J_{r} .
$$

Let $\phi_{I, J}: \Delta^{m-1} \times \Delta^{n-1} \rightarrow \Delta_{I} \times \Delta_{J}$ be the map $\phi_{I} \times \phi_{J}$. Then $\phi_{I, J}$ maps simplices of $\Delta^{m-1} \times \Delta^{n-1}$ containing $\xi$ to simplices of $\Delta_{I} \times \Delta_{J}$ containing $\left\{\bar{e}_{p_{1}} \times \bar{f}_{q_{1}}, \ldots, \bar{e}_{p_{k}} \times\right.$ $\left.\bar{f}_{q_{k}}\right\}$, and this map preserves proper intersections and codimensions. Suppose that $\mathscr{T}$ is a triangulation of $\Delta^{m-1} \times \Delta^{n-1}$ containing $\xi$. Recall that $\mathscr{T}(\xi)$ is the subcollection of simplices of $\mathscr{T}$ containing $\xi$. Then

$$
\phi_{\xi}(\mathscr{T}):=\left\{\phi_{I, J}(\sigma): \sigma \in \mathscr{T}(\xi)\right\}
$$

is a local triangulation of $\Delta_{I} \times \Delta_{J}$ at $\left\{\bar{e}_{p_{1}} \times \bar{f}_{q_{1}}, \ldots, \bar{e}_{p_{k}} \times \bar{f}_{q_{k}}\right\}$. Moreover, $\phi_{I, J}$ gives a bijection between $\mathscr{T}(\xi)$ and $\phi_{\xi}(\mathscr{T})$. If $\mathscr{T}$ is a local triangulation at $\zeta$ and $\xi \cup \zeta \in \mathscr{T}$, then $\phi_{\xi}(\mathscr{T})$ is a local triangulation of $\Delta_{I} \times \Delta_{J}$ at $\left\{\bar{e}_{p_{1}} \times \bar{f}_{q_{1}}, \ldots, \bar{e}_{p_{k}} \times \bar{f}_{q_{k}}\right\} \cup \phi_{I, J}(\zeta)$, and $\phi_{I, J}$ gives a bijection between $\mathscr{T}(\xi)$ and $\phi_{\xi}(\mathscr{T})$.

We can use contraction to prove the following useful fact.

Proposition 3.3 Let $\mathscr{T}$ be a local triangulation at $\xi$. Let $\tau \in \mathscr{T}^{*}$, and let $e_{i} \times f_{j} \in$ $\tau \backslash \xi$. Let $\sigma=\tau \backslash\left\{e_{i} \times f_{j}\right\}$. If both connected components of $T(\sigma)$ contain an edge, then there is some $\tau^{\prime} \in \mathscr{T}^{*}$ with $\tau^{\prime}=\sigma \cup\left\{e_{i^{\prime}} \times f_{j^{\prime}}\right\}$, where $e_{i^{\prime}}$ and $f_{j^{\prime}}$ are in different components of $T(\sigma)$ than $e_{i}$ and $f_{j}$, respectively.

Proof Since $e_{i} \times f_{j} \notin \xi, \sigma \in \mathscr{T}$. Let $I=\left(I_{1}, I_{2}\right), J=\left(J_{1}, J_{2}\right)$ be partitions associated to $\sigma$ with $e_{i} \in I_{1}$ and $f_{j} \in J_{2}$. Then $\phi_{\sigma}(\mathscr{T})$ is a local triangulation of $\Delta_{I} \times \Delta_{J}$ at $\bar{\xi}:=\left\{\bar{e}_{1} \times \bar{f}_{1}, \bar{e}_{2} \times \bar{f}_{2}\right\}$. By Proposition 3.1, we have $\bar{\xi} \cup\left\{\bar{e}_{2} \times \bar{f}_{1}\right\} \in \phi_{\sigma}(\mathscr{T})$. Let $\tau^{\prime} \in \mathscr{T}^{*}$ be such that $\phi_{I, J}\left(\tau^{\prime}\right)=\bar{\xi} \cup\left\{\bar{e}_{2} \times \bar{f}_{1}\right\}$. This $\tau^{\prime}$ satisfies the desired conclusion.

\section{Tools}

With the general theory established, we now put them to use to develop the machinery needed in the proof of the theorem.

\subsection{Orders Defined by Triangulations}

In this section we will define several orders given by a triangulation $\mathscr{T}$. Our main tools will be the restriction and contraction operations defined earlier and the characterization of triangulations of $\Delta^{1} \times \Delta^{n-1}$.

\subsubsection{The Restriction Order $\leq_{i_{1} i_{2}}$}

We first consider triangulations induced on $\left(\Delta^{m-1}\right)^{1} \times \Delta^{n-1}$, where $\left(\Delta^{m-1}\right)^{1}$ denotes the 1-skeleton of $\Delta^{m-1}$. These triangulations were first considered by Ardila and Ceballos who completely characterized them when $m=3$ [2]. 
Let $i_{1}, i_{2} \in[m]$ be distinct, and let $I=\left\{e_{i_{1}}, e_{i_{2}}\right\}$. Let $\mathscr{T}$ be a local triangulation of $\Delta^{m-1} \times \Delta^{n-1}$ at $\xi \subseteq I \times \Delta^{n-1}$. The restriction $\mathscr{T}\left[I \times \Delta^{n-1}\right]$ is a local triangulation of $I \times \Delta^{n-1}$ at $\xi$. By mapping $e_{i_{1}} \mapsto e_{1}$ and $e_{i_{2}} \mapsto e_{2}$, we obtain a local triangulation of $\Delta^{1} \times \Delta^{n-1}$. This triangulation and the order (3.2) give a quasiorder on $\Delta^{n-1}$. We denote this order by $\leq \mathscr{T}\left[i_{1} i_{2}\right]$, or simply $\leq_{i_{1} i_{2}}$ if $\mathscr{T}$ is understood. If $\mathscr{T}$ is a triangulation, then $\leq_{i_{1} i_{2}}$ is a total order.

We have the following immediate consequence of Proposition 3.2.

Proposition 4.1 Let $f_{j}, f_{j^{\prime}} \in \Delta^{m-1}$ be distinct. Then $f_{j}<_{i_{1} i_{2}} f_{j^{\prime}}$ if and only if

$$
\left\{e_{i_{2}} \times f_{j}, e_{i_{1}} \times f_{j^{\prime}}\right\} \subseteq \sigma \in \mathscr{T}
$$

for some $\sigma$.

\subsubsection{The Partial Order $\preceq_{i}$}

The next order we will construct is a partial order on the set of all maximal simplices of $\Delta^{m-1} \times \Delta^{n-1}$. It tells us if we can go from one maximal simplex to another along a path that locally "tends toward" the $e_{i}$ direction.

Remark 4.2 F. Santos (private communication, 2016) notes that $\preceq_{i}$ can be understood geometrically as follows: Let $V$ be the real inner product space with orthonormal basis $\Delta^{m-1} \cup \Delta^{n-1}$, and embed $\Delta^{m-1} \times \Delta^{n-1} \subset V$ by $e_{i} \times f_{j} \mapsto e_{i}+f_{j}$. Let $W \subset V$ be the subspace orthogonal to $\sum_{k=1}^{m} e_{k}$ and $\sum_{k=1}^{n} f_{k}$; then $\Delta^{m-1} \times \Delta^{n-1}$ is a full-dimensional polytope in an affine subspace parallel to $W$. Let $u=e_{i}-(1 /(m-1)) \sum_{k \neq i} e_{k} \in W$. Then the covering relations of $\preceq_{i}$ are given by pairs $\tau \prec_{i} \tau^{\prime}$ such that $\tau$ and $\tau^{\prime}$ are adjacent maximal simplices and if $v$ is the normal vector to $\tau \cap \tau^{\prime}$ in $W$ oriented toward $\tau^{\prime}$, then the inner product of $u$ and $v$ is positive.

Note that we can perform the above construction for any triangulation of a point set and vector $u$. However, the result will not in general be a partial order.

Suppose $\tau, \tau^{\prime}$, are two adjacent maximal simplices of $\Delta^{m-1} \times \Delta^{n-1}$. We can write

$$
\tau=\sigma \cup\left\{e_{i} \times f_{j}\right\}, \quad \tau^{\prime}=\sigma \cup\left\{e_{i^{\prime}} \times f_{j^{\prime}}\right\},
$$

where $\sigma$ is the common facet of $\tau, \tau^{\prime}$. Since $\sigma$ has codimension 1 and is not contained in a face of $\Delta^{m-1} \times \Delta^{n-1}, T(\sigma)$ has exactly two connected components, both of which contain an edge. Let $I=\left(I_{1}, I_{2}\right)$ and $J=\left(J_{1}, J_{2}\right)$ be partitions associated to $\sigma$, as defined in Sect. 3.3. There is a unique way to choose $I$ and $J$ so that $e_{i} \times f_{j} \in I_{1} \times J_{2}$ (and thus $e_{i^{\prime}} \times f_{j^{\prime}} \in I_{2} \times J_{1}$ by Proposition 3.3.) In this case, we write

$$
\tau \stackrel{I_{1}, I_{2}}{\longrightarrow} \tau^{\prime}
$$

Now, fix some $i \in[m]$. If $\tau, \tau^{\prime}$ are any two maximal simplices of $\Delta^{m-1} \times \Delta^{n-1}$, we write $\tau \preceq_{i} \tau^{\prime}$ if there is a sequence of maximal simplices $\tau_{1}, \ldots, \tau_{N}$ such that

$$
\tau=\tau_{1} \stackrel{I_{1}^{1}, I_{2}^{1}}{\longrightarrow} \tau_{2} \stackrel{I_{1}^{2}, I_{2}^{2}}{\longrightarrow} \cdots \stackrel{I_{1}^{N-1}, I_{2}^{N-1}}{\longrightarrow} \tau_{N}=\tau^{\prime}
$$


for some partitions $\left(I_{1}^{r}, I_{2}^{r}\right)$ such that $e_{i} \in I_{2}^{r}$ for all $r=1, \ldots, N-1$.

Proposition 4.3 The relation $\preceq_{i}$ is a partial order over the set of maximal simplices of $\Delta^{m-1} \times \Delta^{n-1}$.

Proof We induct on $m$. If $m=1$, then $\Delta^{0} \times \Delta^{n-1}$ has only one maximal simplex and the result follows. Suppose $m>1$. We first note the following.

Proposition 4.4 Suppose $\tau \stackrel{I_{1}, I_{2}}{\longrightarrow} \tau^{\prime}$ and let $e_{i} \in I_{2}$. Then $N_{\tau}\left(e_{i}\right) \subseteq N_{\tau^{\prime}}\left(e_{i}\right)$. Moreover, for any $f_{j} \in N_{\tau}\left(e_{i}\right), N_{\tau}\left(f_{j}\right) \supseteq N_{\tau^{\prime}}\left(f_{j}\right)$.

Proof This follows easily from the definition of $\stackrel{I_{1}, I_{2}}{\longrightarrow}$.

Now, suppose the relation $\preceq_{i}$ contains a cycle. Thus there exist $\tau_{1}, \ldots, \tau_{N}$, where $N>1$, such that

$$
\tau_{1} \stackrel{I_{1}^{1}, I_{2}^{1}}{\longrightarrow} \tau_{2} \stackrel{I_{1}^{2}, I_{2}^{2}}{\longrightarrow} \cdots \stackrel{I_{1}^{N-1}, I_{2}^{N-1}}{\longrightarrow} \tau_{N} \stackrel{I_{1}^{N}, I_{2}^{N}}{\longrightarrow} \tau_{1}
$$

and $e_{i} \in I_{2}^{r}$ for all $r$. Let $f_{j} \in N_{\tau_{1}}\left(e_{i}\right)$ be such that $\left|N_{\tau_{1}}\left(f_{j}\right)\right|>1$; since $T\left(\tau_{1}\right)$ is a spanning tree and $m>1$, such an $f_{j}$ must exist. Let $e_{i^{\prime}} \in N_{\tau_{1}}\left(f_{j}\right) \backslash\left\{e_{i}\right\}$. Since $\tau_{1}, \ldots, \tau_{N}$ is a cycle in $\preceq_{i}$, Proposition 4.4 implies that $f_{j} \in N_{\tau_{r}}\left(e_{i}\right)$ and $e_{i^{\prime}} \in N_{\tau_{r}}\left(f_{j}\right)$ for $r=1, \ldots, N$. Hence, $\xi:=\left\{e_{i} \times f_{j}, e_{i^{\prime}} \times f_{j}\right\} \subseteq \tau_{r}$ for all $r$. Thus, we have a cycle

$$
\phi_{I, J}\left(\tau_{1}\right) \stackrel{\bar{I}_{1}^{1}, \bar{I}_{2}^{1}}{\longrightarrow} \phi_{I, J}\left(\tau_{2}\right) \stackrel{\bar{I}_{1}^{2}, \bar{I}_{2}^{2}}{\longrightarrow} \cdots \stackrel{\bar{I}_{1}^{N-1}, \bar{I}_{2}^{N-1}}{\longrightarrow} \phi_{I, J}\left(\tau_{N}\right) \stackrel{\bar{I}_{1}^{N}, \bar{I}_{2}^{N}}{\longrightarrow} \phi_{I, J}\left(\tau_{1}\right)
$$

in $\Delta_{I} \times \Delta_{J}$, where $I$ and $J$ are partitions associated to $\xi$ and $\bar{I}_{b}^{r}:=\phi_{I}\left(I_{b}^{r}\right)$. Since $\phi_{I}\left(e_{i}\right) \in \bar{I}_{2}^{r}$ for all $r$ and $|I|=m-1$, this contradicts the inductive hypothesis, completing the proof.

We conclude this subsection by sketching how this order can be used to find flips in triangulations of $\Delta^{2} \times \Delta^{n-1}$. This leads to a proof of flip-connectivity of $\Delta^{2} \times \Delta^{n-1}$ which is essentially the same as the proof given by Santos in [14], but stated more concisely. Given a triangulation $\mathscr{T}$ of $\Delta^{2} \times \Delta^{n-1}$, let $\mathscr{T}^{\circ}$ be the set of all maximal simplices $\tau \in \mathscr{T}^{*}$ such that $\left\{e_{1}, e_{2}\right\} \in \operatorname{shape}(\tau)$. We leave the following proposition as an exercise to the reader.

Proposition 4.5 Suppose $\mathscr{T}^{\circ}$ is nonempty, and let $\tau$ be a maximal element of $\mathscr{T}^{\circ}$ with respect to $\preceq_{3}$. Without loss of generality, assume $N_{\tau}\left(f_{1}\right)=\left\{e_{1}, e_{2}\right\}$ and $N_{\tau}\left(f_{2}\right)=$ $\left\{e_{i}, e_{3}\right\}$, where $i=1$ or 2 . Then $\mathscr{T}$ has a flip supported on $\left(\left\{e_{3} \times f_{1}, e_{i} \times f_{2}\right\},\left\{e_{i} \times\right.\right.$ $\left.\left.f_{1}, e_{3} \times f_{2}\right\}\right)$.

\subsubsection{The Quasiorder $\preceq_{i_{1} i_{2}}$}

The partial order in the previous section is unfortunately not enough to find flips in triangulations of $\Delta^{3} \times \Delta^{n-1}$. We will need to use a slightly more complicated order. We will define the order $\preceq_{i_{1} i_{2}}$ on the maximal simplices of a fixed triangulation. It 
will happen that $\tau \preceq_{i_{1} i_{2}} \tau^{\prime}$ if $\tau \preceq_{i_{2}} \tau^{\prime}$. However, we will also have $\tau \preceq_{i_{1} i_{2}} \tau^{\prime}$ if we can move from $\tau$ to $\tau^{\prime}$ along a path which is "purely" in the $e_{i_{1}}$ or $-e_{i_{1}}$ direction. The result is a quasiorder with some equivalencies between simplices.

Remark 4.6 The geometric interpretation of $\preceq_{i_{1} i_{2}}$ is analogous to the one in Remark 4.2 except that we use the direction vector $u=e_{i_{2}}-(1 /(m-2)) \sum_{k \neq i_{1}, i_{2}} e_{k}$. If $\tau$ and $\tau^{\prime}$ are adjacent maximal simplices and $u$ is parallel to $\tau \cap \tau^{\prime}$, then $\tau$ and $\tau^{\prime}$ are equivalent under this order.

Let $i_{1}, i_{2} \in[m]$ be distinct. Let $\mathscr{T}$ be a local triangulation of $\Delta^{m-1} \times \Delta^{n-1}$ at $\left\{e_{i_{2}}\right\} \times J_{0}$ for some (possibly empty) $J_{0} \subseteq \Delta^{n-1}$. Given two maximal simplices $\tau, \tau^{\prime} \in \mathscr{T}^{*}$, we write $\tau \preceq_{i_{1} i_{2}} \tau^{\prime}$ if there is a sequence $\tau_{1}, \ldots, \tau_{N} \in \mathscr{T}^{*}$ such that

$$
\tau=\tau_{1} \stackrel{I_{1}^{1}, I_{2}^{1}}{\longrightarrow} \tau_{2} \stackrel{I_{1}^{2}, I_{2}^{2}}{\longrightarrow} \cdots \stackrel{I_{1}^{N-1}, I_{2}^{N-1}}{\longrightarrow} \tau_{N}=\tau^{\prime}
$$

for some partitions $\left(I_{1}^{r}, I_{2}^{r}\right)$ such that for each $r=1, \ldots, N-1$, either

(A) $e_{i_{2}} \in I_{2}^{r}$, or

(B) $\left\{I_{1}^{r}, I_{2}^{r}\right\}=\left\{\left\{e_{i_{1}}\right\}, \Delta^{m-1} \backslash\left\{e_{i_{1}}\right\}\right\}$.

Then $\preceq_{i_{1} i_{2}}$ is a quasiorder on $\mathscr{T}^{*}$. We wish to determine the elements which are equivalent under $\preceq_{i_{1} i_{2}}$. We denote this equivalence by $\sim_{i_{1} i_{2}}$.

Lemma 4.7 Let $\tau, \tau^{\prime} \in \mathscr{T}^{*}$. We have $\tau \sim_{i_{1} i_{2}} \tau^{\prime}$ if and only if there is $\sigma \in \tau \cap \tau^{\prime}$ such that $T(\sigma)$ has a connected component containing $\Delta^{m-1} \backslash\left\{e_{i_{1}}\right\}$.

Proof First assume that such a $\sigma$ exists. Let $I, J$ be partitions associated to $\sigma$ such that $I=\left(\left\{e_{i_{1}}\right\}, \Delta^{m-1} \backslash\left\{e_{i_{1}}\right\}\right)$. Then $\phi_{I, J}(\tau)$ and $\phi_{I, J}\left(\tau^{\prime}\right)$ are both maximal simplices in $\phi_{\sigma}(\mathscr{T})$. By Proposition 3.1, we thus have a sequence

$$
\phi_{I, J}(\tau)=\tau_{1} \stackrel{I_{1}^{1}, I_{2}^{1}}{\longrightarrow} \tau_{2} \stackrel{I_{1}^{2}, I_{2}^{2}}{\longrightarrow} \cdots \stackrel{I_{1}^{N-1}, I_{2}^{N-1}}{\longrightarrow} \tau_{N}=\phi_{I, J}\left(\tau^{\prime}\right)
$$

where $\tau_{1}, \ldots, \tau_{n} \in \phi_{\sigma}(\mathscr{T})$ and $\left\{I_{1}^{r}, I_{2}^{r}\right\}=\left\{\left\{\bar{e}_{1}\right\},\left\{\bar{e}_{2}\right\}\right\}$ for all $r$. Lifting this sequence to $\mathscr{T}$, we obtain a sequence of adjacent maximal simplices where each adjacency has the form (B). Reversing this sequence also gives a sequence of this form. Hence $\tau \sim_{i_{1} i_{2}} \tau^{\prime}$.

We now prove the converse. Suppose that $\tau_{1}, \ldots, \tau_{N} \in \mathscr{T}^{*}$ are such that

$$
\tau_{1} \stackrel{I_{1}^{1}, I_{2}^{1}}{\longrightarrow} \tau_{2} \stackrel{I_{1}^{2}, I_{2}^{2}}{\longrightarrow} \cdots \stackrel{I_{1}^{N-1}, I_{2}^{N-1}}{\longrightarrow} \tau_{N} \stackrel{I_{1}^{N}, I_{2}^{N}}{\longrightarrow} \tau_{1},
$$

where each adjacency satisfies (A) or (B). We need to prove that there is some $\sigma$ contained in all the $\tau_{r}$ which satisfies the conclusion in the lemma. We induct on $m$. If $m=2$, by Proposition 3.1 there is some $f_{j}$ (namely, the label of the first simplex in the order), such that $e_{i_{2}} \times f_{j} \in \tau$ for any $\tau \in \mathscr{T}^{*}$. We can thus take $\sigma=\left\{e_{i_{2}} \times f_{j}\right\}$.

Assume $m>2$. Recall that $\mathscr{T}$ gives a quasiorder $\leq_{i_{1} i_{2}}$ on $\Delta^{n-1}$. Let $\leq^{1}$ be the "lexicographic-like" quasiorder defined on the power set of $\Delta^{n-1}$ as follows. Let $J=$ $\left\{f_{j_{1}}, \ldots, f_{j_{s}}\right\}$ and $J^{\prime}=\left\{f_{j_{1}^{\prime}}, \ldots, f_{j_{t}^{\prime}}\right\}$ be subsets of $\Delta^{n-1}$ with $f_{j_{1}} \leq_{i_{1} i_{2}} \ldots \leq \leq_{i_{1} i_{2}} f_{j_{s}}$ and $f_{j_{1}^{\prime}} \leq_{i_{1} i_{2}} \ldots \leq_{i_{1} i_{2}} f_{j_{t}^{\prime}}$. Then $J \leq \leq^{1} J^{\prime}$ if either 
(i) $s \geq t$ and $f_{j_{p}}=i_{i_{1} i_{2}} f_{j_{p}^{\prime}}$ for all $p \leq t$, or

(ii) there is some $q \leq s, t$ such that $f_{j_{p}}=i_{i_{1} i_{2}} f_{j_{p}^{\prime}}$ for all $p<q$ and $f_{j_{q}}<_{i_{1} i_{2}} f_{j_{q}^{\prime}}$.

Note that this order is the same as the lexicographic order except that a prefix of a set $J$ comes after $J$ in this order. We have $J<^{1} J^{\prime}$ if and only if (ii) holds or (i) holds and $s>t$. In particular, if $J \supsetneq J^{\prime}$, then $J<^{1} J^{\prime}$.

Given a simplex $\sigma \in \mathscr{T}$, define the sets

$$
R_{\sigma}:=\left\{f_{j} \in N_{\sigma}\left(e_{i_{2}}\right):\left|N_{\sigma}\left(f_{j}\right)\right|>1\right\}
$$

and

$$
S_{\sigma}:=\left\{f_{j} \in N_{\sigma}\left(e_{i_{2}}\right): f_{j} \leq_{i_{1} i_{2}} f_{j^{\prime}} \text { for all } f_{j^{\prime}} \in R_{\sigma}\right\}
$$

Note that if $\sigma$ is maximal, then $R_{\sigma} \cap S_{\sigma}$ is nonempty (specifically, it contains the minimal elements of $R_{\sigma}$ with respect to $\leq_{i_{1} i_{2}}$ ). We will prove the following.

Proposition 4.8 Suppose $\tau, \tau^{\prime} \in \mathscr{T}^{*}$ are such that $\tau \stackrel{I_{1}, I_{2}}{\longrightarrow} \tau^{\prime}$ and $\left(I_{1}, I_{2}\right)$ satisfies (A) or (B). Then $S_{\tau} \geq^{l} S_{\tau^{\prime}}$. If $S_{\tau}={ }^{l} S_{\tau^{\prime}}$, then $R_{\tau} \cap S_{\tau} \supseteq R_{\tau^{\prime}} \cap S_{\tau^{\prime}}$. Also, for any $f_{j} \in N_{\tau}\left(e_{i_{2}}\right), N_{\tau}\left(f_{j}\right) \backslash\left\{e_{i_{1}}\right\} \supseteq N_{\tau^{\prime}}\left(f_{j}\right) \backslash\left\{e_{i_{1}}\right\}$.

Proof Let $\tau=\sigma \cup\left\{e_{i} \times f_{j}\right\}$ and $\tau^{\prime}=\sigma \cup\left\{e_{i^{\prime}} \times f_{j^{\prime}}\right\}$. We consider separately the cases where $\left(I_{1}, I_{2}\right)$ satisfies $(\mathrm{A})$ or $(\mathrm{B})$.

If (A) holds. We have $R_{\sigma} \subseteq R_{\tau}$. Since $N_{\sigma}\left(e_{i_{2}}\right)=N_{\tau}\left(e_{i_{2}}\right)$, we thus have $S_{\sigma} \supseteq S_{\tau}$, and hence $S_{\sigma} \leq S_{\tau}$. Now, if $e_{i^{\prime}} \neq e_{i_{2}}$, then $S_{\tau^{\prime}}=S_{\sigma}$. Otherwise, we have $R_{\tau^{\prime}}=$ $R_{\sigma} \cup\left\{f_{j^{\prime}}\right\}$ and $N_{\tau^{\prime}}\left(e_{i_{2}}\right)=N_{\sigma}\left(e_{i_{2}}\right) \cup\left\{f_{j^{\prime}}\right\}$. If $f_{j^{\prime}}$ is not a minimal element of $R_{\tau^{\prime}}$, then $S_{\tau^{\prime}}=S_{\sigma}$. Otherwise, $S_{\tau^{\prime}}$ consists of $f_{j^{\prime}}$ and all elements of $S_{\sigma}$ which are less than or equivalent to $f_{j^{\prime}}$. In this case, $S_{\tau^{\prime}}<{ }^{1} S_{\sigma}$. In all cases, $S_{\tau^{\prime}} \leq{ }^{1} S_{\sigma} \leq{ }^{1} S_{\tau}$.

Now suppose $S_{\tau^{\prime}}={ }^{1} S_{\tau}$. By the above argument, we must have $S_{\sigma}={ }^{1} S_{\tau}$, which holds above only if $S_{\sigma}=S_{\tau}$. Since $R_{\sigma} \subseteq R_{\tau}$, we thus have $R_{\sigma} \cap S_{\sigma} \subseteq R_{\tau} \cap S_{\tau}$. We must also have $S_{\tau^{\prime}}={ }^{1} S_{\sigma}$, and so either $e_{i^{\prime}} \neq e_{i_{2}}$ or $f_{j^{\prime}}$ is not a minimal element of $R_{\tau^{\prime}}$. In either case, $R_{\tau^{\prime}}$ and $R_{\sigma}$ have the same minimal elements, so $R_{\tau^{\prime}} \cap S_{\tau^{\prime}}=R_{\sigma} \cap S_{\sigma}$. Thus, $R_{\tau^{\prime}} \cap S_{\tau^{\prime}} \subseteq R_{\tau} \cap S_{\tau}$. Also, by Proposition 4.4, for any $f_{j^{\prime \prime}} \in N_{\tau}\left(e_{i_{2}}\right)$ we have $N_{\tau}\left(f_{j^{\prime \prime}}\right) \supseteq N_{\tau^{\prime}}\left(f_{j^{\prime \prime}}\right)$, and thus $N_{\tau}\left(f_{j^{\prime \prime}}\right) \backslash\left\{e_{i_{1}}\right\} \supseteq N_{\tau^{\prime}}\left(f_{j^{\prime \prime}}\right) \backslash\left\{e_{i_{1}}\right\}$.

If $(B)$ holds. If $\left(I_{1}, I_{2}\right)=\left(\left\{e_{i_{1}}\right\}, \Delta^{n-1} \backslash\left\{e_{i_{1}}\right\}\right)$, then we have Case (A). So assume $\left(I_{1}, I_{2}\right)=\left(\Delta^{n-1} \backslash\left\{e_{i_{1}}\right\},\left\{e_{i_{1}}\right\}\right)$. We consider two cases.

Case 1: $R_{\sigma} \neq \emptyset$. Let $f_{j^{*}} \in R_{\sigma}$. Since

$$
\left\{e_{i_{2}} \times f_{j^{*}}, e_{i_{1}} \times f_{j}\right\},\left\{e_{i_{2}} \times f_{j^{*}}, e_{i_{1}} \times f_{j^{\prime}}\right\} \subseteq \tau^{\prime},
$$

by Proposition 4.1 we have $f_{j^{*}}<_{i_{1} i_{2}} f_{j}, f_{j^{\prime}}$. Since $f_{j^{*}}<_{i_{1} i_{2}} f_{j}$, removing $f_{j}$ from $N_{\tau}\left(e_{i_{2}}\right)$ and $R_{\tau}$ does not change $S_{\tau}$ or the set of minimal elements of $R_{\tau}$. Similarly, adding $f_{j^{\prime}}$ to $R_{\sigma}$ does not change $S_{\sigma}$ or the set of minimal elements of $R_{\sigma}$. Thus, $S_{\tau}=S_{\sigma}=S_{\tau^{\prime}}$ and $R_{\tau} \cap S_{\tau}=R_{\sigma} \cap S_{\sigma}=R_{\tau^{\prime}} \cap S_{\tau^{\prime}}$. 
Case 2: $R_{\sigma}=\emptyset$. Then we must have $R_{\tau}=\left\{f_{j}\right\}$ and $R_{\tau^{\prime}}=\left\{f_{j^{\prime}}\right\}$. Then since

$$
\left\{e_{i_{2}} \times f_{j^{\prime}}, e_{i_{1}} \times f_{j}\right\} \subseteq \tau^{\prime}
$$

by Proposition 4.1 we have $f_{j^{\prime}}<_{i_{1} i_{2}} f_{j}$. It follows that $S_{\tau^{\prime}}$ consists of $f_{j^{\prime}}$ and all elements of $S_{\tau}$ that are less than or equivalent to $f_{j^{\prime}}$. Thus $S_{\tau^{\prime}}<S_{\tau}$.

In either case, it is clear that $N_{\tau}\left(f_{j^{\prime \prime}}\right) \backslash\left\{e_{i_{1}}\right\} \supseteq N_{\sigma}\left(f_{j^{\prime \prime}}\right) \backslash\left\{e_{i_{1}}\right\}$ for all $f_{j^{\prime \prime}} \in \Delta^{n-1}$. This completes the proof of the proposition.

We return to the proof of the lemma. Recall that we have a cycle (4.1). By Proposition 4.8, we must have $S_{\tau_{1}}={ }^{1} \cdots={ }^{1} S_{\tau_{N}}$, and thus $R_{\tau_{1}} \cap S_{\tau_{1}}=\cdots=R_{\tau_{N}} \cap S_{\tau_{N}}$. Let $f_{j} \in R_{\tau_{1}} \cap S_{\tau_{1}}$. By Proposition 4.8, $N_{\tau_{r}}\left(f_{j}\right) \backslash\left\{e_{i_{1}}\right\}$ is the same set $N$ for all $r$.

Clearly $e_{i_{2}} \in N$. First suppose $N=\left\{e_{i_{2}}\right\}$. Since $f_{j} \in R_{\tau_{r}}$ for all $r$, we must have $N_{\tau_{r}}\left(f_{j}\right)=\left\{e_{i_{1}}, e_{i_{2}}\right\}$ for all $r$. Hence, none of the adjacencies in (4.1) can satisfy (B). Thus all of them satisfy (A), which means we have a cycle in the order $\preceq_{i_{2}}$. By Proposition 4.3, this means the cycle has one element, in which case the result trivially holds.

Now suppose $N \supsetneq\left\{e_{i_{2}}\right\}$. Then there is some $e_{i} \neq e_{i_{1}}, e_{i_{2}}$ which is in $N_{\tau_{r}}\left(f_{j}\right)$ for all $r$. Thus $\xi:=\left\{e_{i} \times f_{j}, e_{i_{2}} \times f_{j}\right\} \subseteq \tau_{r}$ for all $r$. We obtain a cycle

$$
\phi_{I, J}\left(\tau_{1}\right) \stackrel{\bar{I}_{1}^{1}, \bar{I}_{2}^{1}}{\longrightarrow} \phi_{I, J}\left(\tau_{2}\right) \stackrel{\bar{I}_{1}^{2}, \bar{I}_{2}^{2}}{\longrightarrow} \cdots \stackrel{\bar{I}_{1}^{N-1}, \bar{I}_{2}^{N-1}}{\longrightarrow} \phi_{I, J}\left(\tau_{N}\right) \stackrel{\bar{I}_{1}^{N}, \bar{I}_{2}^{N}}{\longrightarrow} \phi_{I, J}\left(\tau_{1}\right)
$$

where $I$ and $J$ are partitions associated to $\xi$ and $\bar{I}_{b}^{r}:=\phi_{I}\left(I_{b}^{r}\right)$. Each simplex in this cycle belongs to the local triangulation $\phi_{\xi}(\mathscr{T})$ of $\Delta_{I} \times \Delta_{J}$ at $\left\{\phi_{I}\left(e_{i_{2}}\right)\right\} \times \phi_{J}\left(J_{0} \cup\left\{f_{j}\right\}\right)$. Furthermore, all adjacencies satisfy (A) or (B) with $e_{i_{1}}$ and $e_{i_{2}}$ replaced by $\phi_{I}\left(e_{i_{1}}\right)$ and $\phi_{J}\left(e_{i_{2}}\right)$, respectively. Thus, by the inductive hypothesis, there is some $\bar{\sigma}$ contained in all the $\phi_{I, J}\left(\tau_{r}\right)$ such that $T(\bar{\sigma})$ has a connected component containing $\Delta_{I} \backslash\left\{\phi_{I}\left(e_{i_{1}}\right)\right\}$. We can write $\bar{\sigma}=\phi_{I, J}(\sigma)$ for some $\sigma \in \mathscr{T}$ containing $\xi$. This $\sigma$ satisfies the conclusion of the lemma.

\subsection{Structure of Local Triangulations}

Let $\mathscr{T}$ be a local triangulation of $\Delta^{m-1} \times \Delta^{n-1}$ at $\xi$. Let $e_{i_{0}} \times f_{j_{0}} \in \xi$. The main goal of this section is to prove the following.

Proposition 4.9 There is a unique minimal element of $\mathscr{T}^{*}$ with respect to $\preceq_{i_{0}}$.

Let $u, v$ be vertices of $G_{m, n}$. An alternating path (with respect to $\xi$ ) from $u$ to $v$ is a path in $G_{m, n}$ from $u$ to $v$ such that every other edge of the path is an element of $\xi$. A 1-alternating path is an alternating path such that the first, third, fifth, and so on edges are in $\xi$, and a 2-alternating path is an alternating path such that the second, fourth, sixth, and so on edges are in $\xi$. We note the following.

Proposition 4.10 Let $\sigma, \sigma^{\prime} \in \mathscr{T}$, and let $b=1$ or 2 . Suppose there are $b$-alternating paths $P, P^{\prime}$ from u to $v$ in $T(\sigma), T\left(\sigma^{\prime}\right)$, respectively. Then $P=P^{\prime}$. 
Proof By walking along $P$ from $u$ to $v$ and then walking backward along $P^{\prime}$ from $v$ to $u$, we obtain a closed walk. Since $G_{m, n}$ is bipartite and elements of $\xi$ are elements of both $\sigma$ and $\sigma^{\prime}$, this walk alternates between elements of $\sigma$ and elements of $\sigma^{\prime}$. If $P \neq P^{\prime}$, then from this walk we can obtain a cycle which alternates between elements of $\sigma$ and elements of $\sigma^{\prime}{ }^{3}$ Thus $\sigma$ and $\sigma^{\prime}$ contain opposite parts of a circuit, which is impossible for a triangulation.

Proposition 4.11 Let $\tau \in \mathscr{T}^{*}$. Then $\tau$ is minimal in $\mathscr{T}^{*}$ with respect to $\preceq_{i_{0}}$ if and only if for every $e_{i} \in \Delta^{m-1}$, the path in $T(\tau)$ from $e_{i_{0}}$ to $e_{i}$ is 1-alternating.

Proof Suppose $\tau$ is minimal in $\mathscr{T}^{*}$ with respect to $\preceq_{i_{0}}$. Let $e_{i} \in \Delta^{m-1}$, and let $P$ be the path in $T(\tau)$ from $e_{i_{0}}$ to $e_{i}$. Assume $P$ is not 1 -alternating. Then $P$ contains consecutive vertices $e_{i^{\prime}}, f_{j^{\prime}}$ in that order such that $e_{i^{\prime}} \times f_{j^{\prime}} \notin \xi$. By Proposition 3.3, there is some $\tau^{\prime} \in \mathscr{T}^{*}$ with $\tau^{\prime} \stackrel{I_{1}, I_{2}}{\longrightarrow} \tau$ such that $e_{i^{\prime}}, e_{i_{0}} \in I_{2}$. This contradicts the minimality of $\tau$ in $\preceq_{i_{0}}$.

Conversely, suppose that for each $e_{i} \in \Delta^{m-1}$ the path in $T(\tau)$ from $e_{i_{0}}$ to $e_{i}$ is 1-alternating. Suppose that there is some $\tau^{\prime} \in \mathscr{T}^{*}$ with $\tau^{\prime} \stackrel{I_{1}, I_{2}}{\longrightarrow} \tau$, where $e_{i_{0}} \in I_{2}$. Let $\sigma=\tau \cap \tau^{\prime}$, and let $e_{i^{\prime}} \times f_{j^{\prime}}=\tau \backslash \sigma$. Let $e_{i} \in I_{1}$, and consider the path in $T(\tau)$ from $e_{i_{0}}$ to $e_{i}$. Since this path is 1 -alternating and must contain $e_{i^{\prime}} \times f_{j^{\prime}}$, we have $e_{i^{\prime}} \times f_{j^{\prime}} \in \xi$. But $e_{i^{\prime}} \times f_{j^{\prime}} \notin \tau^{\prime}$, contradicting $\tau^{\prime} \in \mathscr{T}$. Thus $\tau$ is minimal in $\mathscr{T}^{*}$ with respect to $\preceq_{i_{0}}$.

We can now prove Proposition 4.9.

Proof of Proposition 4.9 Suppose $\tau, \tau^{\prime}$ are minimal in $\mathscr{T}^{*}$ with respect to $\preceq_{i_{0}}$. Let $\sigma, \sigma^{\prime}$ be the minimal subsets of $\tau, \tau^{\prime}$, respectively, such that $T(\sigma)$ and $T\left(\sigma^{\prime}\right)$ contain $\Delta^{m-1}$. By Propositions 4.11 and 4.10, we have $\sigma=\sigma^{\prime}$. Now, $\phi_{\sigma}(\mathscr{T})$ has a single maximal simplex, and both $\tau$ and $\tau^{\prime}$ must map to this simplex. Hence $\tau=\tau^{\prime}$, as desired.

\subsection{Circuits and Flips}

In this final subsection, we collect some facts about flips. We first prove the following general fact about flips of point configurations.

Proposition 4.12 Let $\mathscr{T}$ be a triangulation of a point set $A$ and let $X=\left\{X^{+}, X^{-}\right\}$be a circuit in $A$, where $X^{+}=\left\{x_{1}, \ldots, x_{k}\right\}$. Suppose that $X^{-} \in \mathscr{T}$. Then $\mathscr{T}$ has a fip supported on $\left(X^{+}, X^{-}\right)$if and only if there is no maximal simplex $\tau \in \mathscr{T}\left(X^{-}\right)^{*}$ with $|\tau \cap X| \leq|X|-2$. If $\mathscr{T}$ does not have a flip supported on $\left(X^{+}, X^{-}\right)$and $X \backslash\left\{x_{i}\right\} \in \mathscr{T}$ for some $i$, then such a $\tau$ exists with $\tau \cap X=X \backslash\left\{x_{i}, x_{j}\right\}$ for some $j \neq i$.

Proof First, suppose that $\mathscr{T}$ has a flip supported on $\left(X^{+}, X^{-}\right)$and such a $\tau$ exists. Since $|\tau \backslash X| \geq|\tau|-|X|+2$ and $\tau$ is maximal, $\tau \backslash X$ is not in the link of any maximal simplex of $\mathscr{T}_{X}^{+}$. Thus, in the collection $\mathscr{T}^{\prime}$ defined in Proposition 2.1, we have $\tau \in \mathscr{T}^{\prime}$ but $X^{-} \notin \mathscr{T}^{\prime}$, contradicting the fact that $\mathscr{T}^{\prime}$ is a triangulation.

Conversely, suppose that $\mathscr{T}$ does not have a flip supported on $\left(X^{+}, X^{-}\right)$. Consider a maximal simplex $\tau \in \mathscr{T}^{*}$ containing $X^{-}$. If $X \backslash\left\{x_{i}\right\} \notin \mathscr{T}$ for all $i$, then $\mid \tau \cap$

3 Again, using bipartiteness. 
$X|\leq| X \mid-2$ and we are done. Otherwise, if $X \backslash\left\{x_{i}\right\} \in \mathscr{T}$, then choose $\tau$ so that $X \backslash\left\{x_{i}\right\} \subseteq \tau$.

Suppose there is no $\tau^{\prime} \in \mathscr{T}\left(X^{-}\right)^{*}$ and $j \neq i$ such that $\tau^{\prime} \cap X=X \backslash\left\{x_{i}, x_{j}\right\}$. Let $j \neq i$. Consider the facet $\sigma:=\tau \backslash\left\{x_{j}\right\}$ of $\tau$. We claim that $\sigma$ is not contained in a face of $\operatorname{conv}(A)$. Assume the contrary, and let $H$ be a supporting hyperplane of this face. Since $\tau$ is a maximal simplex, $x_{j} \notin H$. Then since $x_{i}, x_{j}$ are in $X^{+}$of the circuit $X$ and $X \backslash\left\{x_{i}, x_{j}\right\} \subseteq H$, this implies $x_{i}$ and $x_{j}$ are on opposite sides of $H$. This contradicts the fact that $H$ is a supporting hyperplane. Thus, $\sigma$ is not contained in a face of $\operatorname{conv}(A)$. It follows that there is another maximal simplex $\tau^{\prime} \in \mathscr{T}^{*}$ containing $\sigma$. If $x_{i} \notin \tau^{\prime}$, then $\left|\tau^{\prime} \cap X\right|=X \backslash\left\{x_{i}, x_{j}\right\}$, a contradiction. Thus we have that $\tau^{\prime}=\tau \backslash\left\{x_{j}\right\} \cup\left\{x_{i}\right\}$, and hence $X \backslash\left\{x_{j}\right\} \in \mathscr{T}$ and $\tau \backslash X \in \operatorname{link} \mathscr{T}\left(X \backslash\left\{x_{j}\right\}\right)$. We thus have link $\mathscr{T}\left(X \backslash\left\{x_{i}\right\}\right) \subseteq \operatorname{link}_{\mathscr{T}}\left(X \backslash\left\{x_{j}\right\}\right)$.

Switching $i$ and $j$ in the above argument, we either have $\tau^{\prime} \cap X=X \backslash\left\{x_{j}, x_{i}\right\}$ for some $\tau^{\prime} \in \mathscr{T}\left(X^{-}\right)^{*}$ or link $\mathscr{T}\left(X \backslash\left\{x_{j}\right\}\right) \subseteq \operatorname{link}_{\mathscr{T}}\left(X \backslash\left\{x_{i}\right\}\right)$. Hence link $\mathscr{T}\left(X \backslash\left\{x_{i}\right\}\right)=$ link $\mathscr{T}\left(X \backslash\left\{x_{j}\right\}\right)$. Since this holds for all $j \neq i$, we have $\mathscr{T}_{X}^{+} \subseteq \mathscr{T}$ and every maximal simplex of $\mathscr{T}_{X}^{+}$has the same link in $\mathscr{T}$. Hence $\mathscr{T}$ has a flip supported on $\left(X^{+}, X^{-}\right)$, a contradiction. This completes the proof.

Now, let $\mathscr{T}$ be a triangulation of $\Delta^{m-1} \times \Delta^{n-1}$. Let $X=\left\{X^{+}, X^{-}\right\}$be a circuit of $\Delta^{m-1} \times \Delta^{n-1}$, where

$$
\begin{aligned}
& X^{-}=\left\{e_{i_{1}} \times f_{j_{1}}, e_{i_{2}} \times f_{j_{2}}, \ldots, e_{i_{k}} \times f_{j_{k}}\right\}, \\
& X^{+}=\left\{e_{i_{2}} \times f_{j_{1}}, e_{i_{3}} \times f_{j_{2}}, \ldots, e_{i_{1}} \times f_{j_{k}}\right\} .
\end{aligned}
$$

By Proposition 4.9, for each $r=1, \ldots, k, \mathscr{T}\left(X^{-}\right)^{*}$ has a unique minimal element in the order $\preceq_{i_{r}}$. Denote this element by $\tau_{r}$. For each $r=1, \ldots, k$, let

$$
\sigma_{r}:=X \backslash\left\{e_{i_{r}} \times f_{j_{r-1}}\right\}
$$

be a maximal simplex of $\mathscr{T}_{X}^{+} \cdot 4$

Proposition 4.13 If $\sigma_{r} \in \mathscr{T}$, then $\sigma_{r} \subseteq \tau_{r}$.

Proof We have $\sigma_{r} \in \mathscr{T}\left(X^{-}\right)$. The edges of $T\left(\sigma_{r} \backslash\left\{e_{i_{r-1}} \times f_{j_{r-1}}\right\}\right)$ form a 1-alternating path with respect to $X^{-}$from $e_{i_{r}}$ to $e_{i_{r+1}}, e_{i_{r+2}}, \ldots, e_{i_{r-1}}$. So by Propositions 4.11 and 4.10, $\sigma_{r} \backslash\left\{e_{i_{r-1}} \times f_{j_{r-1}}\right\} \subseteq \tau_{r}$. Finally, $e_{i_{r-1}} \times f_{j_{r-1}} \in \tau_{r}$ because $X^{-} \subseteq \tau_{r}$.

Now assume that $\mathscr{T}$ has a flip supported on $\left(X^{+}, X^{-}\right)$. Let $\mathscr{T}^{\prime}$ be the result of this flip. We will prove the following two propositions. They will later be used to determine the effect that certain flips have on the shapes of the simplices involved.

Proposition 4.14 Let $e_{i} \in \Delta^{m-1}$ where $i \neq i_{1}, \ldots, i_{k}$. Then there is a unique $j \in$ $\left\{j_{1}, \ldots, j_{k}\right\}$ such that $\sigma_{r} \cup\left\{e_{i} \times f_{j}\right\} \in \mathscr{T}$ for some $r$.

Proof We first show that such a $j$ exists. By Proposition 4.11, there is a 1-alternating path with respect to $X^{-}$from $e_{i_{1}}$ to $e_{i}$ in $T\left(\tau_{1}\right)$. Since $i \neq i_{1}, \ldots, i_{k}$, the last edge of

\footnotetext{
${ }^{4}$ Here, as in the rest of the section, subscripts of $i, j$, and $\sigma$ are taken modulo $k$.
} 
this path must be of the form $e_{i} \times f_{j}$ for some $j \in\left\{j_{1}, \ldots, j_{k}\right\}$. Thus $\left\{e_{i} \times f_{j}\right\} \subseteq \tau_{1}$, and so by Proposition 4.13, $\sigma_{1} \cup\left\{e_{i} \times f_{j}\right\} \in \mathscr{T}$.

Now suppose a different such $j^{\prime}$ exists, and let $\sigma_{r}$ be such that $\sigma_{r} \cup\left\{e_{i} \times f_{j^{\prime}}\right\} \in \mathscr{T}$. By Proposition 2.1, $\sigma_{r}$ and $\sigma_{1}$ have the same link in $\mathscr{T}$, so we may assume $\sigma_{r}=\sigma_{1}$. Then there is a 1-alternating path from $e_{i_{1}}$ to $e_{i}$ in $T\left(\sigma_{1} \cup\left\{e_{i} \times f_{j}\right\}\right)$ and a different 1alternating path from $e_{i_{1}}$ to $e_{i}$ in $T\left(\sigma_{1} \cup\left\{e_{i} \times f_{j^{\prime}}\right\}\right)$. This contradicts Proposition 4.10. Hence $j$ is unique.

Proposition 4.15 There is a bijection $\Psi: \mathscr{T}^{*} \rightarrow\left(\mathscr{T}^{\prime}\right)^{*}$ such that for each $\tau \in \mathscr{T}^{*}$ and $f_{j} \in \Delta^{n-1}$, we have

1. $N_{\Psi(\tau)}\left(f_{j}\right)=N_{\tau}\left(f_{j}\right)$ if $j \neq j_{1}, \ldots, j_{k}$.

2. If $j=j_{r}$, then either $N_{\Psi(\tau)}\left(f_{j}\right)=N_{\tau}\left(f_{j}\right)$ or

$$
N_{\Psi(\tau)}\left(f_{j}\right)=N_{\tau}\left(f_{j}\right) \backslash\left\{e_{i_{r}}\right\} \cup\left\{e_{i_{r+1}}\right\},
$$

with the latter occurring if and only if $\sigma_{r+1}=X \backslash\left\{e_{i_{r+1}} \times f_{j_{r}}\right\} \subseteq \tau$.

Proof Let $\tau \in \mathscr{T}^{*}$. By Proposition 4.12, either $\tau$ does not contain $X^{-}$or $\tau$ contains a maximal simplex of $\mathscr{T}_{X}^{+}$. In the former case, define $\Psi(\tau)=\tau$. Otherwise, suppose $\sigma_{r+1} \subseteq \tau$ for some $r$. Define

$$
\Psi(\tau)=\tau \backslash\left\{e_{i_{r}} \times f_{j_{r}}\right\} \cup\left\{e_{i_{r+1}} \times f_{j_{r}}\right\}
$$

By Proposition $2.1, \Psi$ is a bijection $\mathscr{T}^{*}$ to $\left(\mathscr{T}^{\prime}\right)^{*}$, and it satisfies the desired properties.

Finally, we note the following relation between flips and the restriction order $\leq_{i_{1} i_{2}}$.

Proposition 4.16 Let $\mathscr{T}, X$, and $\mathscr{T}^{\prime}$ be as above. Let $i, i^{\prime}$ be distinct elements of $[m]$. If $\left|X^{-}\right| \neq 2$ or $\left|X^{-}\right|=2$ and $\left\{i, i^{\prime}\right\} \neq\left\{i_{1}, i_{2}\right\}$, then the orders $\leq \mathscr{T}\left[i i^{\prime}\right]$ and $\leq \mathscr{T}^{\prime}\left[i i^{\prime}\right]$ are the same. If $\left|X^{-}\right|=2$ and $\left\{i, i^{\prime}\right\}=\left\{i_{1}, i_{2}\right\}$, then the orders $\leq \mathscr{T}\left[i i^{\prime}\right]$ and $\leq \mathscr{T}^{\prime}\left[i i^{\prime}\right]$ are the same except the order of the consecutive elements $f_{j_{1}}, f_{j_{2}}$ is flipped.

Proof This follows easily from Propositions 4.15 and 4.1.

\section{The Main Proof}

We are now ready to prove the main result.

Theorem 5.1 The set of triangulations of $\Delta^{3} \times \Delta^{n-1}$ is flip-connected.

Our proof will be an algorithm that starts with any triangulation of $\Delta^{3} \times \Delta^{n-1}$ and applies flips to reach a specific triangulation. The algorithm will have three "phases". Phase I is much, much more difficult than the other two, and for the sake of page length, it is the only phase whose proof is included in this paper. The other two proofs can be found in the pre-print version [8]. 
Remark 5.2 For readers familiar with the Cayley trick, we can interpret the phases as follows. Consider the mixed subdivision $\mathscr{S}$ of $n \Delta^{3}$ corresponding to the given triangulation. Phase I moves the cells of $\mathscr{S}$ so that all cells which are unmixed simplices or triangular prisms of shape $\left\{\left\{e_{1}, e_{2}, e_{4}\right\}\right\}$ are adjacent to the face $n\left\{e_{1}, e_{2}, e_{3}\right\}$ of $n \Delta^{3}$. Phase II then moves all the unmixed simplices to the edge $n\left\{e_{1}, e_{2}\right\}$. Finally, Phase III permutes the unmixed simplices and sorts out the remaining cells.

For notational convenience, we will now refer to $e_{i}$ as simply $i$.

\subsection{Phase I}

Let $\mathscr{T}$ be a triangulation of $\Delta^{3} \times \Delta^{n-1}$. Let $\mathscr{T}^{\mathrm{I}}$ be the set of all maximal simplices $\tau \in \mathscr{T}^{*}$ for which there is some $f_{j} \in \Delta^{n-1}$ with $\{1,2\} \subseteq N_{\tau}\left(f_{j}\right)$ and $4 \notin N_{\tau}\left(f_{j}\right)$. The goal of Phase I is to prove the following.

Claim 5.3 $\mathscr{T}$ is flip-connected to a triangulation $\mathscr{T}^{\prime}$ with $\left(\mathscr{T}^{\prime}\right)^{\mathrm{I}}=\emptyset$.

Assume $\mathscr{T}^{\mathrm{I}} \neq \varnothing$. To prove the claim, it suffices to show that $\mathscr{T}$ is flip-connected to some $\mathscr{T}^{\prime}$ with $\left|\left(\mathscr{T}^{\prime}\right)^{\mathrm{I}}\right|<\left|\mathscr{T}^{\mathrm{I}}\right|$.

We will first need to consider how certain flips on $\mathscr{T}$ change $\mathscr{T}^{\mathrm{I}}$. In all of the below propositions, $X$ is as in (4.2).

Proposition 5.4 Suppose that $\mathscr{T}$ has a flip supported on $\left(X^{+}, X^{-}\right)$, and let $\mathscr{T}^{\prime}$ be the result of this flip. Assume that $1=i_{1}$ and $2 \notin\left\{i_{1}, \ldots, i_{k}\right\}$. Suppose that there is a maximal simplex $\sigma$ of $\mathscr{T}_{X}^{+}$and $j \in\left\{j_{1}, \ldots, j_{k}\right\}$ such that $\sigma \cup\left\{2 \times f_{j}\right\} \in \mathscr{T}$. We have the following.

(a) If $j \neq j_{k}$, then $\left|\left(\mathscr{T}^{\prime}\right)^{\mathrm{I}}\right| \leq\left|\mathscr{T}^{\mathrm{I}}\right|$.

(b) If $j=j_{1}$ and $4 \in\left\{i_{1}, \ldots, i_{k}\right\}$, then $\left|\left(\mathscr{T}^{\prime}\right)^{\mathrm{I}}\right|<\left|\mathscr{T}^{\mathrm{I}}\right|$.

Proof Let $\Psi$ be as in Proposition 4.15. Suppose $N_{\Psi(\tau)}\left(f_{j^{\prime}}\right) \supseteq\{1,2\}$ for some $\Psi(\tau) \in$ $\mathscr{T}^{\prime}$ and $f_{j^{\prime}} \in \Delta^{n-1}$. Then by Proposition 4.15, either $N_{\tau}\left(f_{j^{\prime}}\right)=N_{\Psi(\tau)}\left(f_{j^{\prime}}\right)$ or $j^{\prime}=j_{k}$ and $\sigma_{1} \cup\left\{2 \times f_{j_{k}}\right\} \subseteq \tau$. In the latter case, Proposition 4.14 implies $j=j_{k}$. Thus if $j \neq j_{k},\left|\left(\mathscr{T}^{\prime}\right)^{\mathrm{I}}\right| \leq\left|\bar{T}^{\mathrm{I}}\right|$. If furthermore $j=j_{1}$, then

$$
\mathscr{S}:=\left\{\tau \in \mathscr{T}^{*}: \sigma_{2} \cup\left\{2 \times f_{j_{1}}\right\} \subseteq \tau\right\}
$$

is nonempty. If furthermore $4 \in\left\{i_{1}, \ldots, i_{k}\right\}$, then $4 \notin N_{\tau}\left(f_{j_{1}}\right)$ for any $\tau \in \mathscr{S}$, so $\mathscr{S} \subseteq \mathscr{T}^{\mathrm{I}}$. But $\Psi(\mathscr{S}) \cap\left(\mathscr{T}^{\prime}\right)^{\mathrm{I}}=\emptyset$, so $\left|\left(\mathscr{T}^{\prime}\right)^{\mathrm{I}}\right|<\left|\mathscr{T}^{\mathrm{I}}\right|$.

Note that we could swap the roles of 1 and 2 in the above proposition and the result would still hold. The following propositions are proved similarly; we leave them to the reader.

Proposition 5.5 Suppose that $\mathscr{T}$ has a flip supported on $\left(X^{+}, X^{-}\right)$, and let $\mathscr{T}^{\prime}$ be the result of this flip. Assume that $1,2 \notin\left\{i_{1}, \ldots, i_{k}\right\}$ and $4=i_{2}$. Suppose there is a maximal simplex $\sigma$ of $\mathscr{T}_{X}^{+}$and $j, j^{\prime} \in\left\{j_{1}, \ldots, j_{k}\right\}$ such that $\sigma \cup\left\{1 \times f_{j}, 2 \times f_{j^{\prime}}\right\} \in \mathscr{T}$. We have the following. 
(a) If $j \neq j^{\prime}$, then $\left|\left(\mathscr{T}^{\prime}\right)^{\mathrm{I}}\right|=\left|\mathscr{T}^{\mathrm{I}}\right|$.

(b) If $j=j^{\prime}=j_{1}$, then $\left|\left(\mathscr{T}^{\prime}\right)^{\mathrm{I}}\right|<\left|\mathscr{T}^{\mathrm{I}}\right|$.

Proposition 5.6 Suppose that $\mathscr{T}$ has a flip supported on $\left(X^{+}, X^{-}\right)$, and let $\mathscr{T}^{\prime}$ be the result of this flip. Assume that $1,2 \in\left\{i_{1}, \ldots, i_{k}\right\}$. Then $\left|\left(\mathscr{T}^{\prime}\right)^{\mathrm{I}}\right|=\left|\mathscr{T}^{\mathrm{I}}\right|$.

Now, let $\tau_{\mathrm{I}}$ be any maximal element of $\mathscr{T}^{\mathrm{I}}$ with respect to $\preceq_{34}$. Let $\operatorname{shape}\left(\tau_{\mathrm{I}}\right)=$ $\left\{N_{1}, \ldots, N_{k}\right\}$, and without loss of generality, let $f_{1}, \ldots, f_{k} \in \Delta^{n-1}$ be such that $N_{r}=N_{\tau_{\mathrm{I}}}\left(f_{r}\right)$. Since $\tau_{\mathrm{I}} \in \mathscr{T}^{\mathrm{I}}$, we can assume $\{1,2\} \subseteq N_{1}$ (and thus $4 \notin N_{1}$ ). Without loss of generality, we then have three distinct possibilities for shape $\left(\tau_{\mathrm{I}}\right)$.

1. $\{1,4\}$ or $\{2,4\} \subseteq N_{2}$.

2. $N_{1}=\{1,2\}, N_{2}=\{3,4\}, N_{3}=\{1,3\}$ or $\{2,3\}$.

3. $N_{1}=\{1,2,3\}, N_{2}=\{3,4\}$.

The remainder of Phase I will depend on which case we are in. Each case will use the same strategy, which we outline as follows. Let $\sigma_{\mathrm{I}}$ be the unique minimal subset of $\tau_{\mathrm{I}}$ such that $T\left(\sigma_{\mathrm{I}}\right)$ has a connected component containing $\{1,2,4\}$. Call a subcollection $\mathscr{S} \subseteq \mathscr{T}$ of simplices $\tau_{\mathrm{I}^{-}}$good if both of the following hold.

(a) There is no maximal simplex $\tau \in \mathscr{S}$ such that $\tau \in \mathscr{T}^{\mathrm{I}}$ and $\sigma_{\mathrm{I}} \nsubseteq \tau$.

(b) There are no maximal simplex $\tau \in \mathscr{S}$ and $j \neq 1,2$ such that $\{1,2\} \subseteq N_{\tau}\left(f_{j}\right)$.

The strategy will be to define a circuit $X=\left\{X^{+}, X^{-}\right\}$such that $\mathscr{T}\left(X^{-}\right)$is $\tau_{\mathrm{I}}$-good, and so that if $\mathscr{T}$ has a flip supported on $\left(X^{+}, X^{-}\right)$, then the result of the flip will decrease the size of $\mathscr{T}^{\mathrm{I}}$. Once $X$ is defined, we will use $\tau_{\mathrm{I}}$-goodness to find a series of intermediate flips starting from $\mathscr{T}$ so that the final result has a flip supported on $\left(X^{+}, X^{-}\right)$. (See, for example, Claim 5.8.)

\subsubsection{Case $1:\{1,4\}$ or $\{2,4\} \subseteq N_{2}$}

We will assume $\{1,4\} \subseteq N_{2}$; the other case follows analogously. Let $X=\left\{X^{+}, X^{-}\right\}$ be the circuit

$$
X^{-}=\left\{1 \times f_{1}, 4 \times f_{2}\right\}, \quad X^{+}=\left\{4 \times f_{1}, 1 \times f_{2}\right\} .
$$

Then $\sigma=X \backslash\left\{4 \times f_{1}\right\}$ is a maximal simplex of $\mathscr{T}_{X}^{+}$, and $\sigma_{\mathrm{I}}=\sigma \cup\left\{2 \times f_{1}\right\} \subseteq \tau_{\mathrm{I}}$. Thus, if $\left(X^{+}, X^{-}\right)$supports a flip of $\mathscr{T}$, then this flip satisfies the conditions of Proposition 5.4(b), and we would be done.

Let $\tau_{*}$ be the unique minimal element of $\mathscr{T}\left(X^{-}\right)^{*}$ with respect to $\preceq_{4}$. Note that $\sigma_{\mathrm{I}} \in \mathscr{T}\left(X^{-}\right)$, and the edges of $T\left(\sigma_{\mathrm{I}}\right)$ give a 1-alternating path with respect to $X^{-}$from 4 to 1 and 2. Thus, by Propositions 4.11 and 4.10, we have $\sigma_{\mathrm{I}} \subseteq \tau_{*}$. By Lemma 4.7, it follows that $\tau_{*} \sim_{34} \tau_{\mathrm{I}}$. Thus, $\tau_{*}$ is maximal in $\preceq_{34}$. Moreover, its shape satisfies Case 1. We may thus redefine $\tau_{\mathrm{I}}$ as $\tau_{\mathrm{I}}=\tau_{*}$.

We can now make the following key observation.

Proposition $5.7 \mathscr{T}\left(X^{-}\right)$is $\tau_{\mathrm{I}^{-}}$good. 
Proof First, suppose there is some $\tau \in \mathscr{T}\left(X^{-}\right)^{*}$ such that $\tau \in \mathscr{T}^{\mathrm{I}}$ and $\sigma_{\mathrm{I}} \nsubseteq \tau$. Since $\tau_{\mathrm{I}}$ is minimal in $\mathscr{T}\left(X^{-}\right)^{*}$ with respect to $\preceq_{4}$, we have $\tau_{\mathrm{I}} \preceq_{34} \tau$. On the other hand, $\sigma_{\mathrm{I}} \nsubseteq \tau$, and $\sigma_{\mathrm{I}}$ is the unique minimal subset of $\tau_{\mathrm{I}}$ such that $T\left(\sigma_{\mathrm{I}}\right)$ has a connected component containing $\{1,2,4\}$. Thus, by Proposition 4.7, $\tau_{\mathrm{I}} \nsim_{34} \tau$. Hence $\tau_{\mathrm{I}} \prec_{34} \tau$, which contradicts the maximality of $\tau_{\mathrm{I}}$ in $\mathscr{T}^{\mathrm{I}}$ with respect to $\preceq_{34}$.

Now suppose there are some $\tau \in \mathscr{T}\left(X^{-}\right)^{*}$ and $j \neq 1,2$ such that $\{1,2\} \subseteq N_{\tau}\left(f_{j}\right)$. Since $j \neq 2$, by Proposition 3.3, there is some $\tau^{\prime} \in \mathscr{T}\left(X^{-}\right)$(possibly the same as $\tau$ ) with $N_{\tau^{\prime}}\left(f_{j}\right)=N_{\tau}\left(f_{j}\right) \backslash\{4\}$. Since $\{1,2\} \subseteq N_{\tau^{\prime}}\left(f_{j}\right)$ and $j \neq 1$, we must also have $\sigma_{\mathrm{I}} \nsubseteq \tau^{\prime}$. Thus $\tau^{\prime} \in \mathscr{T}^{\mathrm{I}}$ and $\sigma_{\mathrm{I}} \nsubseteq \tau^{\prime}$, which as above is a contradiction.

It now suffices to prove the following claim.

Claim 5.8 Let $\mathscr{T}$ be any triangulation of $\Delta^{3} \times \Delta^{n-1}$ such that $\tau_{\mathrm{I}} \in \mathscr{T}$ and $\mathscr{T}\left(X^{-}\right)$ is $\tau_{\mathrm{I}}$-good. Then either there is a flip of $\mathscr{T}$ supported on $\left(X^{+}, X^{-}\right)$, or there is a flip of $\mathscr{T}$ with result $\mathscr{T}^{\prime}$ such that $\tau_{\mathrm{I}} \in \mathscr{T}^{\prime}, \mathscr{T}^{\prime}\left(X^{-}\right)$is $\tau_{\mathrm{I}}-\operatorname{good},\left|\left(\mathscr{T}^{\prime}\right)^{\mathrm{I}}\right| \leq\left|\mathscr{T}^{\mathrm{I}}\right|$, and $\left|\mathscr{T}^{\prime}\left(X^{-}\right)^{*}\right|<\left|\mathscr{T}\left(X^{-}\right)^{*}\right|$.

By repeatedly applying this claim to our original $\mathscr{T}$, we eventually obtain that $\mathscr{T}$ is flip-connected to some $\mathscr{T}^{\prime}$ for which $\tau_{\mathrm{I}} \in \mathscr{T}^{\prime},\left|\left(\mathscr{T}^{\prime}\right)^{\mathrm{I}}\right| \leq\left|\mathscr{T}^{\mathrm{I}}\right|$, and $\mathscr{T}^{\prime}$ has a flip supported on $\left(X^{+}, X^{-}\right)$. Then by Proposition 5.4, the result $\mathscr{T}^{\prime \prime}$ of this flip satisfies $\left|\left(\mathscr{T}^{\prime \prime}\right)^{\mathrm{I}}\right|<\left|\mathscr{T}^{\mathrm{I}}\right|$, as desired.

Proof of Claim 5.8 Let $\mathscr{S}$ be the set of all maximal simplices $\tau \in \mathscr{T}\left(X^{-}\right)^{*}$ such that $\tau \cap X^{+}=\emptyset$. If $\mathscr{S}=\emptyset$, then by Proposition 4.12, $\mathscr{T}$ has a flip supported on $\left(X^{+}, X^{-}\right)$, and we are done. Assume $\mathscr{S} \neq \varnothing$.

We will use the following to restrict the possible shapes of simplices in $\mathscr{S}$.

Proposition 5.9 Let $\tau \in \mathscr{S}$. The following are true.

(a) $1 \times f_{2} \notin \tau$,

(b) $4 \times f_{1} \notin \tau$,

(c) $2 \times f_{1} \notin \tau$,

(d) $2 \times f_{2} \notin \tau$.

Proof Parts (a) and (b) hold by the definition of $\mathscr{S}$. Suppose $2 \times f_{1} \in \tau$. By part (b), we have $4 \notin N_{\tau}\left(f_{1}\right)$. Hence $\tau \in \mathscr{T}^{\mathrm{I}}$. By part (a), we have $\sigma_{\mathrm{I}} \nsubseteq \tau$. This contradicts

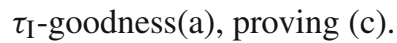

Finally, since $\tau_{\mathrm{I}}$ is minimal in $\mathscr{T}\left(X^{-}\right)^{*}$ with respect to $\preceq_{4}$, by Proposition 4.4 we have $N_{\tau_{\mathrm{I}}}\left(f_{2}\right) \supseteq N_{\tau}\left(f_{2}\right)$. Hence $2 \times f_{2} \notin \tau$, proving $(\mathrm{d})$.

Suppose $\tau \in \mathscr{S}$. Let

$$
P=\left\{1 \times f_{j_{1}}, i_{1} \times f_{j_{1}}, i_{1} \times f_{j_{2}}, i_{2} \times f_{j_{2}}, \ldots, i_{k-1} \times f_{j_{k}}, 4 \times f_{j_{k}}\right\}
$$

be the path in $T(\tau)$ from 1 to 4 . Suppose first that

$$
P=\left\{1 \times f_{j_{1}}, 4 \times f_{j_{1}}\right\} .
$$

By Proposition 5.9(a) and (b), we have $j_{1} \neq 1,2$. Consider the minimal element $\tau^{\prime}$ of $\mathscr{T}\left(X^{-} \cup P\right)^{*}$ with respect to $\preceq_{4}$. Since $P \subseteq \tau^{\prime}$, we have $\tau^{\prime} \in \mathscr{S}$. Now, by 
Proposition 4.11, we must have $2 \times f_{j} \in \tau^{\prime}$ for some $j=1,2$, or $j_{1}$. This contradicts either Proposition $5.9(\mathrm{c}),(\mathrm{d})$, or $\tau_{\mathrm{I}}$-goodness $(\mathrm{b})$. Hence $P$ cannot be of this form.

Furthermore, if $i_{1}=2$, then we have $\{1,2\} \subseteq N_{\tau}\left(f_{j_{1}}\right)$, which contradicts either Proposition 5.9 (c) or $\tau_{\mathrm{I}}$-goodness(b). Hence, $i_{1}=3$.

Now suppose that $f_{j_{k}}=f_{2}$. By Proposition $5.9(\mathrm{~d})$, we have $i_{k-1} \neq 2$. Hence $i_{k-1}=3$, and

$$
P=\left\{1 \times f_{j_{1}}, 3 \times f_{j_{1}}, 3 \times f_{2}, 4 \times f_{2}\right\}
$$

Let $\tau^{\prime}$ be the minimal element of $\mathscr{T}\left(X^{-} \cup P\right)^{*}$ with respect to $\preceq_{4}$. Since $P \subseteq \tau^{\prime}$, we have $\tau^{\prime} \in \mathscr{S}$. By Proposition 4.11, we have $2 \times f_{j} \in \tau^{\prime}$ for some $j=1,2$, or $j_{1}$. As before, this is a contradiction. Hence, $j_{k} \neq 2$.

We have thus restricted $P$ to two possible forms:

(i) $P=\left\{1 \times f_{j_{1}}, 3 \times f_{j_{1}}, 3 \times f_{j_{2}}, 4 \times f_{j_{2}}\right\}, j_{2} \neq 2$.

(ii) $P=\left\{1 \times f_{j_{1}}, 3 \times f_{j_{1}}, 3 \times f_{j_{2}}, 2 \times f_{j_{2}}, 2 \times f_{j_{3}}, 4 \times f_{j_{3}}\right\}, j_{3} \neq 2$.

Now, choose $\tau$ so that it is a maximal element of $\mathscr{S}$ with respect to $\preceq_{21}$. We have two possibilities depending on $P$.

Subcase 1.1: $P$ has form (i). Let $Y=\left\{Y^{+}, Y^{-}\right\}$be the circuit

$$
\begin{aligned}
& Y^{-}=\left\{1 \times f_{j_{1}}, 3 \times f_{j_{2}}, 4 \times f_{2}\right\}, \\
& Y^{+}=\left\{3 \times f_{j_{1}}, 4 \times f_{j_{2}}, 1 \times f_{2}\right\} .
\end{aligned}
$$

Let $\rho=Y \backslash\left\{1 \times f_{2}\right\}$. Then $\rho$ is a maximal simplex of $\mathscr{T}_{Y}^{+}$and $\rho \cup\left\{1 \times f_{1}\right\}=$ $X^{-} \cup P \subseteq \tau$. Let $\tau_{*}$ be the minimal element of $\mathscr{T}\left(X^{-} \cup P\right)^{*}$ with respect to $\preceq_{1}$. Since $P \subseteq \tau_{*}$, we have $\tau_{*} \in \mathscr{S}$. Now, by Proposition 4.11, we must have $2 \times f_{j} \in \tau_{*}$ for some $j=1,2, j_{1}$, or $j_{2}$. We cannot have $j=1,2$, or $j_{1}$ by Propositions 5.9 (c), (d), and $\tau_{\mathrm{I}}$-goodness(b). Thus $2 \times f_{j_{2}} \in \tau_{*}$. Then by Proposition 4.11 , it follows that $\tau_{*}$ is the minimal element of $\mathscr{T}\left(Y^{-}\right)^{*}$ with respect to $\preceq_{1}$.

Since $P \subseteq \tau_{*}$, by Lemma 4.7, we have $\tau \sim_{21} \tau_{*}$. Hence $\tau_{*}$ is a maximal element of $\mathscr{S}$ with respect to $\preceq_{21}$, and $P \subseteq \tau_{*}$. We may thus redefine $\tau$ as $\tau=\tau_{*}$.

We first claim that $\mathscr{T}\left(Y^{-}\right) \subseteq \mathscr{T}\left(X^{-}\right)$. Suppose that $\tau^{\prime} \in \mathscr{T}\left(Y^{-}\right)^{*}$. Clearly $4 \times f_{2} \in$ $\tau^{\prime}$. Also, since $\tau$ is minimal in $\mathscr{T}\left(Y^{-}\right)^{*}$ with respect to $\preceq_{1}$, by Proposition 4.4 we have $N_{\tau}(1) \subseteq N_{\tau^{\prime}}(1)$. Hence $1 \times f_{1} \in \tau^{\prime}$. So $\tau^{\prime} \in \mathscr{T}\left(X^{-}\right)$, as desired.

We now claim that $\mathscr{T}$ has a flip supported on $\left(Y^{+}, Y^{-}\right)$. Suppose the contrary. Since $\rho \in \mathscr{T}$, by Proposition 4.12, there is some $\tau^{\prime} \in \mathscr{T}\left(Y^{-}\right)^{*}$ such that $\left|\tau^{\prime} \cap Y^{+}\right| \leq\left|Y^{+}\right|-$ $2=1$ and $1 \times f_{2} \notin \tau^{\prime}$. As just shown, $\tau^{\prime} \in \mathscr{T}\left(X^{-}\right)$. Moreover, by Proposition 4.4, we have $N_{\tau}\left(f_{1}\right) \supseteq N_{\tau^{\prime}}\left(f_{1}\right)$, so $4 \times f_{1} \notin \tau^{\prime}$. Hence $\tau^{\prime} \in \mathscr{S}$. Since $\left|\tau^{\prime} \cap Y^{+}\right| \leq 1$, we have $P \nsubseteq \tau^{\prime}$, and hence by Lemma 4.7, $\tau \nsim_{21} \tau^{\prime}$. Since $\tau$ is minimal in $\mathscr{T}\left(Y^{-}\right)$with respect to $\preceq_{1}$, we thus have $\tau \prec_{21} \tau^{\prime}$. This contradicts the maximality of $\tau$ in $\mathscr{S}$ with respect to $\preceq_{21}$. Hence, $\mathscr{T}$ has a flip supported on $\left(Y^{+}, Y^{-}\right)$.

Let $\mathscr{T}^{\prime}$ be the result of this flip. By Proposition 4.15, if $j_{1} \neq 1$, we have

$$
\mathscr{T}^{\prime}\left(X^{-}\right)^{*}=\mathscr{T}\left(X^{-}\right)^{*} \backslash \mathscr{T}\left(Y^{-}\right)^{*} \cup \Psi\left(\mathscr{T}\left(X \backslash\left\{3 \times f_{j_{1}}\right\}\right)^{*}\right) \cup \Psi\left(\mathscr{T}\left(X \backslash\left\{4 \times f_{j_{2}}\right\}\right)^{*}\right),
$$

\footnotetext{
${ }^{5}$ Here, as in the rest of the proof, we mean the order $\preceq 21$ which is defined on all of $\mathscr{T}$.
} 
and if $j_{1}=1$, we have

$$
\mathscr{T}^{\prime}\left(X^{-}\right)^{*}=\mathscr{T}\left(X^{-}\right)^{*} \backslash \mathscr{T}\left(Y^{-}\right)^{*} \cup \Psi\left(\mathscr{T}\left(X \backslash\left\{4 \times f_{j_{2}}\right\}\right)^{*}\right) .
$$

Either way, $\left|\mathscr{T}^{\prime}\left(X^{-}\right)^{*}\right|<\left|\mathscr{T}\left(X^{-}\right)^{*}\right|$. By Proposition $5.4(\mathrm{a})$, since $\rho \cup\left\{2 \times f_{j_{2}}\right\} \in$ $\mathscr{T}\left(X^{-}\right)$, we have $\left|\left(\mathscr{T}^{\prime}\right)^{\mathrm{I}}\right| \leq\left|\mathscr{T}^{\mathrm{I}}\right|$. It is easy to check using the above equations that if $\mathscr{T}\left(X^{-}\right)$is $\tau_{\mathrm{I}}$-good, then so is $\mathscr{T}^{\prime}\left(X^{-}\right)$. Finally, to show that $\tau_{\mathrm{I}} \in \mathscr{T}^{\prime}\left(X^{-}\right)$, it suffices to show $\tau_{\mathrm{I}} \notin \mathscr{T}\left(Y^{-}\right)$. If $\tau_{\mathrm{I}} \in \mathscr{T}\left(Y^{-}\right)$, then by Proposition $4.4, N_{\tau}\left(f_{1}\right) \supseteq N_{\tau_{\mathrm{I}}}\left(f_{1}\right)$, so $2 \times f_{1} \notin \tau_{\mathrm{I}}$, a contradiction. Hence $\tau_{\mathrm{I}} \notin \mathscr{T}\left(Y^{-}\right)$, which completes the subcase.

Subcase 1.2: $P$ has form (ii). Let $Y=\left\{Y^{+}, Y^{-}\right\}$be the circuit

$$
\begin{aligned}
& Y^{-}=\left\{1 \times f_{j_{1}}, 3 \times f_{j_{2}}, 2 \times f_{j_{3}}, 4 \times f_{2}\right\}, \\
& Y^{+}=\left\{3 \times f_{j_{1}}, 2 \times f_{j_{2}}, 4 \times f_{j_{3}}, 1 \times f_{2}\right\} .
\end{aligned}
$$

Let $\rho=Y \backslash\left\{1 \times f_{2}\right\}$. Then $\rho$ is a maximal simplex of $\mathscr{T}_{Y}^{+}$and $\rho \subseteq \tau$. By Proposition $4.11, \tau$ is the minimal element of $\mathscr{T}\left(Y^{-}\right)^{*}$ with respect to $\preceq_{1}$.

By the same arguments as in the previous subcase, we have $\mathscr{T}\left(Y^{-}\right) \subseteq \mathscr{T}\left(X^{-}\right)$and $\mathscr{T}$ has a flip supported on $\left(Y^{+}, Y^{-}\right)$. If the result of this flip is $\mathscr{T}^{\prime}$, then Proposition 5.6 implies $\left|\left(\mathscr{T}^{\prime}\right)^{\mathrm{I}}\right|=\left|\mathscr{T}^{\mathrm{I}}\right|$. Similar arguments to the previous subcase show that the rest of Claim 5.8 holds. This concludes Case 1.

\subsubsection{Case $2: N_{1}=\{1,2\}, N_{2}=\{3,4\}, N_{3}=\{1,3\}$ or $\{2,3\}$}

We will assume $N_{3}=\{1,3\}$; the other case follows analogously. Let $X=\left\{X^{+}, X^{-}\right\}$ be the circuit

$$
\begin{aligned}
& X^{-}=\left\{1 \times f_{1}, 4 \times f_{2}, 3 \times f_{3}\right\}, \\
& X^{+}=\left\{4 \times f_{1}, 3 \times f_{2}, 1 \times f_{3}\right\} .
\end{aligned}
$$

Then $\sigma=X \backslash\left\{4 \times f_{1}\right\}$ is a maximal simplex of $\mathscr{T}_{X}^{+}$, and $\sigma_{\mathrm{I}}=\sigma \cup\left\{2 \times f_{1}\right\} \subseteq \tau_{\mathrm{I}}$. Thus, if $\left(X^{+}, X^{-}\right)$supports a flip of $\mathscr{T}$, then this flip satisfies the conditions of Proposition 5.4(b), and we would be done.

By Proposition $4.11, \tau_{\mathrm{I}}$ is minimal in $\mathscr{T}\left(X^{-}\right)^{*}$ with respect to $\preceq_{4}$. We can use the same argument as in Case 1 to prove that $\mathscr{T}\left(X^{-}\right)$is $\tau_{\mathrm{I}}$-good.

It now suffices to prove Claim 5.8 with $\tau_{\mathrm{I}}, \sigma_{\mathrm{I}}$, and $X$ redefined as in this case. Let $\mathscr{S}$ be the set of all maximal simplices $\tau \in \mathscr{T}\left(X^{-}\right)^{*}$ for which $\left|\tau \cap X^{+}\right| \leq 1$ and $4 \times f_{1} \notin \tau$. Since $\sigma \in \mathscr{T}$, by Proposition 4.12 we have that $\left(X^{+}, X^{-}\right)$supports a flip of $\mathscr{T}$ if and only if $\mathscr{S}=\emptyset$. So assume $\mathscr{S} \neq \emptyset$.

Proposition 5.10 Let $\tau \in \mathscr{S}$. The following are true.

(a) $1 \times f_{2} \notin \tau$,

(b) $4 \times f_{1} \notin \tau$,

(c) $2 \times f_{j} \notin \tau$ for $j=1,2,3$. 
Proof Suppose $1 \times f_{2} \in \tau$. Then, with respect to $X^{-}$, we have a 2-alternating path $f_{2} \rightarrow 1$ in $T(\tau)$ and a 2-alternating path $f_{2} \rightarrow 3 \rightarrow f_{3} \rightarrow 1$ in $T\left(\tau_{\mathrm{I}}\right)$. This contradicts Proposition 4.10, proving (a). Part (b) follows from the definition of $\mathscr{S}$.

Suppose $2 \times f_{j} \in \tau$. First suppose $j=1$. By (b), $4 \times f_{1} \notin \tau$, so $\tau \in \mathscr{T}^{\mathrm{I}}$. Since $\left|\tau \cap X^{+}\right| \leq 1$, we have $\sigma_{\mathrm{I}} \not \tau \tau$. This contradicts $\tau_{\mathrm{I}}$-goodness(a). So $j \neq 1$. If $j=2$, then we have, with respect to $X^{-}$, a 2-alternating path $f_{2} \rightarrow 2$ in $T(\tau)$ and a 2-alternating path $f_{2} \rightarrow 3 \rightarrow f_{3} \rightarrow 1 \rightarrow f_{1} \rightarrow 2$ in $T\left(\tau_{\mathrm{I}}\right)$. Thus $j \neq 2$. The same argument using $f_{3} \rightarrow 1 \rightarrow f_{1} \rightarrow 2$ in $T\left(\tau_{\mathrm{I}}\right)$ shows that $j \neq 3$. This proves (c).

Suppose $\tau \in \mathscr{S}$. Let

$$
P=\left\{1 \times f_{j_{1}}, i_{1} \times f_{j_{1}}, i_{1} \times f_{j_{2}}, i_{2} \times f_{j_{2}}, \ldots, i_{k-1} \times f_{j_{k}}, 4 \times f_{j_{k}}\right\}
$$

be the path in $T(\tau)$ from 1 to 4 . By the same arguments as in Case 1, we have $k>1, i_{1}=3$, and $f_{j_{k}} \neq f_{2}$. Furthermore, suppose that $f_{j_{1}} \neq f_{3}$. Let $\tau^{\prime}$ be the minimal element of $\mathscr{T}\left(X^{-} \cup\left\{1 \times f_{j_{1}}, 3 \times f_{j_{1}}\right\}\right)$ with respect to $\preceq_{4}$. Since $\tau^{\prime} \preceq_{4} \tau$, by Propostition 4.4 we have $N_{\tau^{\prime}}(4) \subseteq N_{\tau}(4)$, and hence $4 \times f_{1} \notin \tau^{\prime}$. In addition, $\left\{1 \times f_{j_{1}}, 3 \times f_{j_{1}}, 3 \times f_{3}\right\} \subseteq \tau^{\prime}$ and $f_{j_{1}} \neq f_{3}$, so $1 \times f_{3} \notin \tau^{\prime}$. Thus, $\tau^{\prime} \in \mathscr{S}$. Now, by Proposition 4.11, we must have $2 \times f_{j} \in \tau^{\prime}$ for some $j=1,2,3$, or $j_{1}$. However, all of these cases contradict Proposition 5.10(c) or $\tau_{\mathrm{I}}$-goodness(b). Hence $j_{1}=3$.

It follows that $P$ must sing.

(i) $P=\left\{1 \times f_{3}, 3 \times f_{3}, 3 \times f_{j_{2}}, 4 \times f_{j_{2}}\right\}, j_{2} \neq 2$,

(ii) $P=\left\{1 \times f_{3}, 3 \times f_{3}, 3 \times f_{j_{2}}, 2 \times f_{j_{2}}, 2 \times f_{j_{3}}, 4 \times f_{j_{3}}\right\}, j_{3} \neq 2$.

Now, choose $\tau$ to be a maximal element of $\mathscr{S}$ with respect to $\preceq_{24}$. We consider Subcases (i) and (ii).

Subcase 2.1: $P$ has form (i). Let $Y=\left\{Y^{+}, Y^{-}\right\}$be the circuit

$$
\begin{aligned}
& Y^{-}=\left\{1 \times f_{1}, 4 \times f_{j_{2}}, 3 \times f_{3}\right\} \\
& Y^{+}=\left\{4 \times f_{1}, 3 \times f_{j_{2}}, 1 \times f_{3}\right\} .
\end{aligned}
$$

Let $\rho=Y \backslash\left\{4 \times f_{1}\right\}$. Then $\rho$ is a maximal simplex of $\mathscr{T}_{Y}^{+}$and $\rho \cup\left\{4 \times f_{2}\right\}=$ $X^{-} \cup P \subseteq \tau$. Let $\tau_{*}$ be the minimal element of $\mathscr{T}\left(X^{-} \cup P\right)$ with respect to $\preceq_{4}$. Since $P \subseteq \tau_{*}$, we have $\tau_{*} \in \mathscr{S}$. By Proposition 4.11, we have $2 \times f_{j} \in \tau_{*}$ for some $j=1,2,3$, or $j_{2}$. By Proposition 5.10 (c), we thus have $2 \times j_{2} \in \tau_{*}$. It follows by Proposition 4.11 that $\tau_{*}$ is the minimal element of $\mathscr{T}\left(Y^{-}\right)^{*}$ with respect to $\preceq_{4}$.

Since $P \subseteq \tau_{*}$, by Lemma 4.7, we have $\tau \sim_{24} \tau_{*}$. Hence $\tau_{*}$ is a maximal element of $\mathscr{S}$ with respect to $\preceq_{24}$, and $P \subseteq \tau_{*}$. We may thus redefine $\tau$ as $\tau=\tau_{*}$.

We first claim that $\mathscr{T}\left(Y^{-}\right) \subseteq \mathscr{T}\left(X^{-}\right)$. Suppose that $\tau^{\prime} \in \mathscr{T}\left(Y^{-}\right)^{*}$. Clearly $1 \times f_{1}$, $3 \times f_{3} \in \tau^{\prime}$. Since $\tau$ is minimal in $\mathscr{T}\left(Y^{-}\right)^{*}$ with respect to $\preceq_{4}$, by Proposition 4.4 we have $N_{\tau}(4) \subseteq N_{\tau^{\prime}}(4)$. Hence $4 \times f_{2} \in \tau^{\prime}$. So $\tau^{\prime} \in \mathscr{T}\left(X^{-}\right)$.

We now claim that $\mathscr{T}$ has a flip supported on $\left(Y^{+}, Y^{-}\right)$. Suppose the contrary. Since $\rho \in \mathscr{T}$, by Proposition 4.12, there is some $\tau^{\prime} \in \mathscr{T}\left(Y^{-}\right)^{*}$ such that $\left|\tau^{\prime} \cap Y^{+}\right| \leq\left|Y^{+}\right|-$ $2=1$ and $4 \times f_{1} \notin \tau^{\prime}$. As just shown, $\tau^{\prime} \in \mathscr{T}\left(X^{-}\right)$. Moreover, by Proposition 4.4, we have $N_{\tau}\left(f_{2}\right) \supseteq N_{\tau^{\prime}}\left(f_{2}\right)$, so $3 \times f_{2} \notin \tau^{\prime}$. Hence $\tau^{\prime} \in \mathscr{S}$. Since $\left|\tau^{\prime} \cap Y^{+}\right| \leq 1$, we 
have $P \nsubseteq \tau^{\prime}$, and hence by Lemma 4.7, $\tau \nsim_{24} \tau^{\prime}$. Thus, $\tau \prec_{24} \tau^{\prime}$. This contradicts the maximality of $\tau$ in $\mathscr{S}$ with respect to $\preceq 24$. Hence $\mathscr{T}$ has a flip supported on $\left(Y^{+}, Y^{-}\right)$. Let $\mathscr{T}^{\prime}$ be the result of this flip. We have

$$
\mathscr{T}^{\prime}\left(X^{-}\right)^{*}=\mathscr{T}\left(X^{-}\right)^{*} \backslash \mathscr{T}\left(Y^{-}\right)^{*} \cup \Psi\left(\mathscr{T}\left(X \backslash\left\{3 \times f_{j_{2}}\right\}\right)^{*}\right)
$$

Hence $\left|\mathscr{T}^{\prime}\left(X^{-}\right)^{*}\right|<\left|\mathscr{T}\left(X^{-}\right)^{*}\right|$. By Proposition 5.4, since $\rho \cup\left\{2 \times f_{j_{2}}\right\}$, we have $\left|\left(\mathscr{T}^{\prime}\right)^{\mathrm{I}}\right| \leq\left|\mathscr{T}^{\mathrm{I}}\right|$. It is easy to check from the above equation that if $\mathscr{T}\left(X^{-}\right)$is $\tau_{\mathrm{I}}$-good, then so is $\mathscr{T}^{\prime}\left(X^{-}\right)$. Finally, if $\tau_{\mathrm{I}} \in \mathscr{T}\left(Y^{-}\right)$, then as in the previous paragraph we would have $3 \times f_{2} \notin \tau_{\mathrm{I}}$, a contradiction. So $\tau_{\mathrm{I}} \notin \mathscr{T}\left(Y^{-}\right)$, and hence $\tau_{\mathrm{I}} \in \mathscr{T}^{\prime}\left(X^{-}\right)$.

Subcase 2.2: $P$ has form (ii). Let $Y=\left\{Y^{+}, Y^{-}\right\}$be the circuit

$$
\begin{aligned}
& Y^{-}=\left\{1 \times f_{1}, 4 \times f_{j_{3}}, 2 \times f_{j_{2}}, 3 \times f_{3}\right\}, \\
& Y^{+}=\left\{4 \times f_{1}, 2 \times f_{j_{3}}, 3 \times f_{j_{2}}, 1 \times f_{3}\right\} .
\end{aligned}
$$

Let $\rho=Y \backslash\left\{4 \times f_{1}\right\}$. Then $\rho$ is a maximal simplex of $\mathscr{T}_{Y}^{+}$and $\rho \subseteq \tau$. By Proposition $4.11, \tau$ is the minimal element of $\mathscr{T}\left(Y^{-}\right)^{*}$ with respect to $\preceq_{4}$.

By the same arguments as in the previous subcase, we have $\mathscr{T}\left(Y^{-}\right) \subseteq \mathscr{T}\left(X^{-}\right)$and $\mathscr{T}$ has a flip supported on $\left(Y^{+}, Y^{-}\right)$. If the result of this flip is $\mathscr{T}^{\prime}$, then Proposition 5.6 implies $\left|\left(\mathscr{T}^{\prime}\right)^{\mathrm{I}}\right|=\left|\mathscr{T}^{\mathrm{I}}\right|$. Similar arguments to the previous subcase show that the rest of Claim 5.8 holds. This concludes Case 2.

\subsubsection{Case 3: $N_{1}=\{1,2,3\}, N_{2}=\{3,4\}$}

This is the most complicated of the cases. Unlike the previous cases, we will need to find a second "special circuit" after the first. To help the reader, we have sectioned the argument into several parts.

Reduction to Claim 5.13 (Flipping $X$ ). Let $X=\left\{X^{+}, X^{-}\right\}$be the circuit

$$
\begin{aligned}
& X^{-}=\left\{3 \times f_{1}, 4 \times f_{2}\right\}, \\
& X^{+}=\left\{4 \times f_{1}, 3 \times f_{2}\right\} .
\end{aligned}
$$

Let $\sigma=X \backslash\left\{4 \times f_{1}\right\}$. Then $\sigma$ is a maximal simplex of $\mathscr{T}_{X}^{+}$, and $\sigma_{\mathrm{I}}=\sigma \cup\left\{1 \times f_{1}, 2 \times\right.$ $\left.f_{1}\right\} \subseteq \tau_{\text {I }}$. Thus, if $\left(X^{+}, X^{-}\right)$supports a flip of $\mathscr{T}$, then this flip satisfies the conditions of Proposition $5.5(\mathrm{~b})$, and we would be done.

By Proposition $4.11, \tau_{\mathrm{I}}$ is minimal in $\mathscr{T}\left(X^{-}\right)^{*}$ with respect to $\preceq_{4}$. We can use the same argument as in Case 1 to prove that $\mathscr{T}\left(X^{-}\right)$is $\tau_{\text {I-good. }}$

Let

$$
\begin{aligned}
& \mathscr{T}\left(X^{-}\right)_{1}=\left\{\tau \in \mathscr{T}\left(X^{-}\right)^{*}: 2 \times f_{1} \in \tau, 1 \times f_{1} \notin \tau, X^{+} \cap \tau=\emptyset\right\}, \\
& \mathscr{T}\left(X^{-}\right)_{2}=\left\{\tau \in \mathscr{T}\left(X^{-}\right)^{*}: 1 \times f_{1} \in \tau, 2 \times f_{1} \notin \tau, X^{+} \cap \tau=\emptyset\right\} .
\end{aligned}
$$

We will prove the following. 
Proposition 5.11 Let $\mathscr{T}$ be any triangulation of $\Delta^{m-1} \times \Delta^{n-1}$ such that $\tau_{\mathrm{I}} \in \mathscr{T}$ and $\mathscr{T}\left(X^{-}\right)$is $\tau_{\mathrm{I}^{-}}$good. If $\mathscr{T}$ does not have a flip supported on $\left(X^{+}, X^{-}\right)$, then $\mathscr{T}\left(X^{-}\right)_{1}$ and $\mathscr{T}\left(X^{-}\right)_{2}$ are not both empty.

Proof We first note the following.

Proposition 5.12 Let $\mathscr{T}$ be as above. Let $\tau \in \mathscr{T}\left(X^{-}\right)^{*}$. Suppose that $\tau \cap X^{+}=\emptyset$. The following are true.

(a) $1 \times f_{2}, 2 \times f_{2} \notin \tau$.

(b) $\left\{1 \times f_{1}, 2 \times f_{1}\right\} \nsubseteq \tau$.

Proof Since $\tau_{\mathrm{I}}$ is minimal in $\mathscr{T}\left(X^{-}\right)^{*}$ with respect to $\preceq_{4}$, by Proposition 4.4 we have $N_{\tau_{\mathrm{I}}}\left(f_{2}\right) \supseteq N_{\tau}\left(f_{2}\right)$. This proves (a).

Now suppose $\left\{1 \times f_{1}, 2 \times f_{1}\right\} \subseteq \tau$. Since $\tau \cap X^{+}=\emptyset$, it follows that $\tau \in \mathscr{T}^{\mathrm{I}}$ and $\sigma_{\mathrm{I}} \nsubseteq \tau$. This contradicts $\tau_{\mathrm{I}}$-goodness(a). This proves (b).

Now suppose $\mathscr{T}$ does not have a flip supported on $\left(X^{+}, X^{-}\right)$. By Proposition 4.12, there is some $\tau \in \mathscr{T}\left(X^{-}\right)^{*}$ with $\tau \cap X^{+}=\emptyset$. Let

$$
P=\left\{3 \times f_{j_{1}}, i_{1} \times f_{j_{1}}, i_{1} \times f_{j_{2}}, i_{2} \times f_{j_{2}}, \ldots, i_{k-1} \times f_{j_{k}}, 4 \times f_{j_{k}}\right\}
$$

be the path from 3 to 4 in $T(\tau)$. If $k>2$ and $\left\{i_{1}, i_{2}\right\}=\{1,2\}$, then we have $\{1,2\} \subseteq$ $N_{\tau}\left(f_{j_{2}}\right)$ and $j_{2} \neq 1,2$. This contradicts $\tau_{\mathrm{I}}$-goodness(b). Hence, $P$ has one of the following forms:

$$
\begin{aligned}
& P=\left\{3 \times f_{j_{1}}, 4 \times f_{j_{1}}\right\}, \text { or } \\
& P=\left\{3 \times f_{j_{1}}, i_{1} \times f_{j_{1}}, i_{1} \times f_{j_{2}}, 4 \times f_{j_{2}}\right\} .
\end{aligned}
$$

In either case, let $\tau^{\prime}$ be the minimal element of $\mathscr{T}\left(X^{-} \cup P\right)^{*}$ with respect to $\preceq_{4}$. Since $P \subseteq \tau^{\prime}$, we have $\tau^{\prime} \cap X^{+}=\emptyset$.

Suppose $P$ is of the first form. By Proposition 4.11, we have $1 \times f_{j}, 2 \times f_{j^{\prime}} \in \tau^{\prime}$ for some $j, j^{\prime}=1,2$, or $j_{1}$. By Proposition 5.12(a), we have $j, j^{\prime} \neq 2$. If $j=j^{\prime}=1$, this contradicts Proposition 5.12(b). If $j=j^{\prime}=j_{1}$, this contradicts $\tau_{\text {I-goodness(b). }}$ Hence, exactly one of $j, j^{\prime}$ is 1 and the other is $j_{1}$. If $j^{\prime}=1$ and $j=j_{1}$, then $\tau^{\prime} \in \mathscr{T}\left(X^{-}\right)_{1}$. Otherwise, $\tau^{\prime} \in \mathscr{T}\left(X^{-}\right)_{2}$, as desired.

Now suppose $P$ is of the second form. First assume $i_{1}=1$. By Proposition 4.11, we have $2 \times f_{j} \in \tau^{\prime}$ for some $j=1,2, j_{1}$, or $j_{2}$. If $j \in\left\{j_{1}, j_{2}\right\}$ and $j \neq 1,2$, then we have a contradiction by $\tau_{\mathrm{I}}$-goodness(b). Also, Proposition 5.12(a) implies $j \neq 2$. Hence $j=1$. Since we proved $j \neq j_{1}$, we have also shown that $j_{1} \neq 1$. (Also, since $1 \times f_{j_{2}} \in P$, we have $j_{2} \neq 2$ by Proposition 5.12(a).) Hence $\tau^{\prime} \in \mathscr{T}\left(X^{-}\right)_{1}$. By an analogous argument, if $i_{1}=2$ then $\tau^{\prime} \in \mathscr{T}\left(X^{-}\right)_{2}$.

To finish this case, it suffices to prove the following.

Claim 5.13 Let $\mathscr{T}$ be any triangulation of $\Delta^{m-1} \times \Delta^{n-1}$ such that $\tau_{\mathrm{I}} \in \mathscr{T}$ and $\mathscr{T}\left(X^{-}\right)$is $\tau_{\mathrm{I}^{-}}$good. Suppose that $\mathscr{T}\left(X^{-}\right)_{1}$ is nonempty. Then $\mathscr{T}$ is flip-connected to a triangulation $\mathscr{T}^{\prime}$ such that $\tau_{\mathrm{I}} \in \mathscr{T}^{\prime}, \mathscr{T}^{\prime}\left(X^{-}\right)$is $\tau_{\mathrm{I}}$ good, $\left|\left(\mathscr{T}^{\prime}\right)^{\mathrm{I}}\right| \leq\left|\mathscr{T}^{\mathrm{I}}\right|$, $\left|\mathscr{T}^{\prime}\left(X^{-}\right)_{2}\right| \leq\left|\mathscr{T}\left(X^{-}\right)_{2}\right|$, and $\left|\mathscr{T}^{\prime}\left(X^{-}\right)\right|<\left|\mathscr{T}\left(X^{-}\right)\right|$. 
Using this claim, we can apply flips to our original triangulation $\mathscr{T}$ to eventually reach some $\mathscr{T}^{\prime}$ with $\left|\left(\mathscr{T}^{\prime}\right)^{\mathrm{I}}\right| \leq\left|\mathscr{T}^{\mathrm{I}}\right|,\left|\mathscr{T}^{\prime}\left(X^{-}\right)_{2}\right| \leq\left|\mathscr{T}\left(X^{-}\right)_{2}\right|$, and $\mathscr{T}^{\prime}\left(X^{-}\right)_{1}=\varnothing$. By symmetry, the claim also holds after switching the roles of 1 and 2 . Thus, we can show $\mathscr{T}^{\prime}$ is flip-connected to some $\mathscr{T}^{\prime \prime}$ with $\left|\left(\mathscr{T}^{\prime \prime}\right)^{\mathrm{I}}\right| \leq\left|\mathscr{T}^{\mathrm{I}}\right|$ and $\mathscr{T}^{\prime \prime}\left(X^{-}\right)_{1}=\mathscr{T}^{\prime \prime}\left(X^{-}\right)_{2}=$ $\emptyset$. Moreover, the claim implies $\tau_{\mathrm{I}} \in \mathscr{T}^{\prime \prime}$ and $\mathscr{T}^{\prime \prime}\left(X^{-}\right)$is $\tau_{\mathrm{I}}$-good. By Proposition 5.11, it follows that $\mathscr{T}^{\prime \prime}$ has a flip supported on $\left(X^{+}, X^{-}\right)$, and by Proposition $5.5(\mathrm{~b})$, this finishes the case.

Reduction to Claim 5.14 (Flipping $Y$ ). We now prove Claim 5.13. Let $\tau_{0}$ be a maximal element of $\mathscr{T}\left(X^{-}\right)_{1}$ with respect to $\preceq_{14}$. We will use $\tau_{0}$ to find a set of " $\tau_{0}$-good" simplices, in the same way we used $\tau_{\mathrm{I}}$.

Let $P$ be the path from 3 to 4 in $T\left(\tau_{0}\right)$. By the proof of Proposition 5.11, $P$ satisfies one of the following. ${ }^{6}$

(i) $P=\left\{3 \times f_{j_{1}}, 4 \times f_{j_{1}}\right\}, j_{1} \neq 1,2$.

(ii) $P=\left\{3 \times f_{j_{2}}, 1 \times f_{j_{2}}, 1 \times f_{j_{1}}, 4 \times f_{j_{1}}\right\}, j_{2} \neq 1, j_{1} \neq 2$.

We will deal with these subcases simultaneously. Let $\sigma_{0}$ be the unique minimal subset of $\tau_{0}$ such that $T\left(\sigma_{0}\right)$ has a connected component containing $\{2,3,4\}$. Call a collection $\mathscr{S} \subseteq \mathscr{T}$ of simplices $\tau_{0}$-good if there is no maximal simplex $\tau \in \mathscr{S}$ such that $\tau \in \mathscr{T}\left(X^{-}\right)_{1}$ and $\sigma_{0} \nsubseteq \tau$.

Now, if we have Case (i), let $Y=\left\{Y^{+}, Y^{-}\right\}$be the circuit

$$
\begin{aligned}
& Y^{-}=\left\{3 \times f_{1}, 4 \times f_{j_{1}}\right\}, \\
& Y^{+}=\left\{4 \times f_{1}, 3 \times f_{j_{1}}\right\} .
\end{aligned}
$$

If we have Case (ii), let $Y=\left\{Y^{+}, Y^{-}\right\}$be the circuit

$$
\begin{aligned}
& Y^{-}=\left\{3 \times f_{1}, 4 \times f_{j_{1}}, 1 \times f_{j_{2}}\right\}, \\
& Y^{+}=\left\{4 \times f_{1}, 1 \times f_{j_{1}}, 3 \times f_{j_{2}}\right\} .
\end{aligned}
$$

In either case, let $\rho=Y \backslash\left\{4 \times f_{1}\right\}$. Since $\tau_{0} \in \mathscr{T}\left(X^{-}\right)_{1}$, we have $2 \times f_{1} \in \tau_{0}$. Hence $\sigma_{0}=\rho \cup\left\{2 \times f_{1}\right\}$, and $\sigma_{0} \cup\left\{4 \times f_{2}\right\} \subseteq \tau_{0}$.

In Case (ii), we have by Proposition 4.11 that $\tau_{0}$ is the minimal element of $\mathscr{T}\left(Y^{-}\right)$ with respect to $\preceq_{4}$. Suppose we have Case (i). Let $\tau_{*}$ be the minimal element of $\mathscr{T}\left(\sigma_{0} \cup\left\{4 \times f_{2}\right\}\right)$ with respect to $\preceq_{4}$. By the proof of Proposition 5.11, since $2 \times f_{1} \in \tau_{*}$, we have $1 \times f_{j_{1}} \in{ }_{*}$ and $\tau_{*} \in \mathscr{T}\left(X^{-}\right)_{1}$. By Proposition 4.11, it follows that $\tau_{*}$ is minimal in $\mathscr{T}\left(Y^{-}\right)$with respect to $\preceq_{4}$. Also, since $\sigma_{0} \subseteq \tau_{*}$, by Lemma 4.7 we have $\tau_{0} \sim_{14} \tau_{*}$. Hence $\tau_{*}$ is a maximal element of $\mathscr{T}\left(X^{-}\right)_{1}$ with respect to $\preceq_{14}$, and $P \subseteq \tau_{*}$. We may thus redefine $\tau_{0}$ as $\tau_{0}=\tau_{*}$.

In either case, since $\tau_{0}$ is minimal in $\mathscr{T}\left(Y^{-}\right)$with respect to $\preceq_{4}$, the same argument as in the previous cases shows that $\mathscr{T}\left(Y^{-}\right)$is $\tau_{0}$-good.

We now reach our final claim.

\footnotetext{
6 We have $i_{1}=1$ in Case (ii) by the definition of $\mathscr{T}\left(X^{-}\right)_{1}$. Also, notice that we have switched $j_{2}$ and $j_{1}$ in Case (ii) from the original notation; this will allow us later to talk about both cases simultaneously.
} 
Claim 5.14 Let $\mathscr{T}$ be any triangulation of $\Delta^{m-1} \times \Delta^{n-1}$ such that $\tau_{\mathrm{I}}, \tau_{0} \in \mathscr{T}$, $\mathscr{T}\left(X^{-}\right)$is $\tau_{\mathrm{I}^{-}}$good, and $\mathscr{T}\left(Y^{-}\right)$is $\tau_{0^{-}}$good. Then either there is a flip of $\mathscr{T}$ supported on $\left(Y^{+}, Y^{-}\right)$, or there is a flip of $\mathscr{T}$ with result $\mathscr{T}^{\prime}$ such that

(a) $\tau_{I}, \tau_{0} \in \mathscr{T}^{\prime}$,

(b) $\mathscr{T}^{\prime}\left(X^{-}\right)$is $\tau_{\mathrm{I}^{-}}$good,

(c) $\mathscr{T}^{\prime}\left(Y^{-}\right)$is $\tau_{0}$-good,

(d) $\left|\left(\mathscr{T}^{\prime}\right)^{\mathrm{I}}\right| \leq\left|\mathscr{T}^{\mathrm{I}}\right|$,

(e) $\left|\mathscr{T}^{\prime}\left(X^{-}\right)_{2}\right| \leq\left|\mathscr{T}\left(X^{-}\right)_{2}\right|$,

(f) $\left|\mathscr{T}^{\prime}\left(X^{-}\right)^{*}\right| \leq\left|\mathscr{T}\left(X^{-}\right)^{*}\right|$,

(g) $\left|\mathscr{T}^{\prime}\left(Y^{-}\right)^{*}\right|<\left|\mathscr{T}\left(Y^{-}\right)^{*}\right|$.

Let us see how this will complete the proof of Case 3. Suppose $\mathscr{T}$ satisfies (a)(c). We first show that $\mathscr{T}\left(Y^{-}\right) \subseteq \mathscr{T}\left(X^{-}\right)$and $\tau_{\mathrm{I}} \notin \mathscr{T}^{\prime}\left(Y^{-}\right)$. For the first claim, let $\tau \in \mathscr{T}\left(Y^{-}\right)$. Clearly $3 \times f_{1} \in \tau$. Also, by Proposition $4.11, \tau_{0}$ is minimal in $\mathscr{T}\left(Y^{-}\right)$with respect to $\preceq_{4}$. Hence by Proposition 4.4 , we have $N_{\tau_{0}}$ (4) $\subseteq N_{\tau}(4)$, so $4 \times f_{2} \in \tau$. Thus $\tau \in \mathscr{T}\left(X^{-}\right)$, as desired. For the second claim, if $\tau_{\mathrm{I}} \in \mathscr{T}\left(Y^{-}\right)$, then by Proposition $4.4 N_{\tau_{0}}\left(f_{2}\right) \supseteq N_{\tau_{\mathrm{I}}}\left(f_{2}\right)$, so $3 \times f_{2} \notin \tau_{\mathrm{I}}$, a contradiction. Hence $\tau_{\mathrm{I}} \notin \mathscr{T}\left(Y^{-}\right)$.

Now, assuming Claim 5.14, $\mathscr{T}$ is flip-connected to a triangulation $\mathscr{T}^{\prime}$ that satisfies (a)-(f) of the claim and such that there is a flip of $\mathscr{T}^{\prime}$ supported on $\left(Y^{+}, Y^{-}\right)$. Let $\mathscr{T}^{\prime \prime}$ be the result of this flip. In Case (i), we have

$$
\mathscr{T}^{\prime \prime}\left(X^{-}\right)^{*}=\mathscr{T}^{\prime}\left(X^{-}\right)^{*} \backslash \mathscr{T}^{\prime}\left(Y^{-}\right)^{*} \cup \Psi\left(\mathscr{T}^{\prime}\left(Y \backslash\left\{3 \times f_{j_{1}}\right\}\right)^{*}\right)
$$

and in Case (ii), we have

$$
\begin{aligned}
\mathscr{T}^{\prime \prime}\left(X^{-}\right)^{*}= & \mathscr{T}^{\prime}\left(X^{-}\right)^{*} \backslash \mathscr{T}^{\prime}\left(Y^{-}\right)^{*} \cup \Psi\left(\mathscr{T}^{\prime}\left(Y \backslash\left\{1 \times f_{j_{1}}\right\}\right)^{*}\right) \\
& \cup \Psi\left(\mathscr{T}^{\prime}\left(Y \backslash\left\{3 \times f_{j_{2}}\right\}\right)^{*}\right) .
\end{aligned}
$$

In both cases, $\left|\mathscr{T}^{\prime \prime}\left(X^{-}\right)^{*}\right|<\left|\mathscr{T}^{\prime}\left(X^{-}\right)^{*}\right|$. We now check the rest of the conclusions of Claim 5.13. By Proposition 5.5 (a) in Case (i) and Proposition 5.4 (a) in Case (ii), we have $\left|\left(\mathscr{T}^{\prime \prime}\right)^{\mathrm{I}}\right| \leq\left|\left(\mathscr{T}^{\prime}\right)^{\mathrm{I}}\right|$. It is easy to check from the above equations that if $\mathscr{T}^{\prime}\left(X^{-}\right)$is $\tau_{\mathrm{I}}$-good, then so is $\mathscr{T}^{\prime \prime}\left(X^{-}\right)$. As shown above, $\tau_{\mathrm{I}} \notin \mathscr{T}^{\prime}\left(Y^{-}\right)$, so $\tau_{\mathrm{I}} \in \mathscr{T}^{\prime \prime}$. Finally, it is easy to check that if $\Psi(\tau) \in \mathscr{T}^{\prime \prime}\left(X^{-}\right)_{2}$, then $\tau \in \mathscr{T}^{\prime}\left(X^{-}\right)_{2}$. Hence, $\left|\mathscr{T}^{\prime \prime}\left(X^{-}\right)_{2}\right| \leq\left|\mathscr{T}^{\prime}\left(X^{-}\right)_{2}\right|$. This proves Claim 5.13.

It now suffices to prove Claim 5.14.

Proof of Claim 5.14 Suppose $\mathscr{T}$ does not have a flip supported on $\left(Y^{+}, Y^{-}\right)$. Let $\mathscr{S}$ be the set of maximal simplices $\tau \in \mathscr{T}\left(Y^{-}\right)^{*}$ such that $\left|\tau \cap Y^{+}\right| \leq\left|Y^{+}\right|-2$ and $4 \times f_{1} \notin$. Since $\rho \in \mathscr{T}$, by Proposition $4.12, \mathscr{S} \neq \varnothing$.

As shown above, $\tau_{0}$ is the minimal element of $\mathscr{T}\left(Y^{-}\right)^{*}$ with respect to $\preceq_{4}$ and $\mathscr{T}\left(Y^{-}\right) \subseteq \mathscr{T}\left(X^{-}\right)$. We also prove the following. 
Proposition 5.15 Let $\tau \in \mathscr{S}$. The following are true.

(a) $\tau \cap X^{+}=\emptyset$.

(b) $2 \times f_{j_{r}} \notin \tau$ for all $r$.

(c) $i \times f_{j} \notin \tau$ for all $i, j \in\{1,2\}$.

Proof By definition of $\mathscr{S}$, we have $4 \times f_{1} \notin \tau$. Since $\tau_{0}$ is minimal in $\mathscr{T}\left(Y^{-}\right)$with respect to $\preceq_{4}$, by Proposition 4.4 we have $N_{\tau_{0}}\left(f_{2}\right) \supseteq N_{\tau}\left(f_{2}\right)$, so $3 \times f_{2} \notin \tau_{\mathrm{I}}$. This proves (a).

Suppose $2 \times f_{j_{r}} \in \tau$. Then with respect to $Y^{-}$, we have a 2 -alternating path $f_{j_{r}} \rightarrow 2$ in $T(\tau)$, and a 2-alternating path $f_{j_{r}} \rightarrow \cdots \rightarrow 3 \rightarrow f_{1} \rightarrow 2$ in $T\left(\tau_{0}\right)$. This contradicts Proposition 4.10, which proves (b).

By part (a) and Proposition 5.12(a), we have $i \times f_{2} \notin \tau$ for $i=1$, 2. Suppose that $1 \times f_{1} \in \tau$. Let $\tau^{\prime}$ be the minimal element of $\mathscr{T}\left(X^{-} \cup Y^{-} \cup\left\{1 \times f_{1}\right\}\right)$ with respect to $\preceq_{4}$. By Proposition 4.11, we have $2 \times f_{j}$ for some $j=1,2$, or $j_{r}$. This contradicts Propositions 5.12(b), (a), and 5.15(b) respectively. Thus $1 \times f_{1} \notin \tau$.

Finally, suppose that $2 \times f_{1} \in \tau$. As above, we have $1 \times f_{1} \notin \tau$. Hence $\tau \in \mathscr{T}\left(X^{-}\right)_{1}$. But since $\left|\tau \cap Y^{+}\right| \leq\left|Y^{+}\right|-2$, we have $\sigma_{0} \nsubseteq \tau$, contradicting $\tau_{0}$-goodness. This proves $(\mathrm{c})$.

Now, let

$$
\mathscr{S}^{\prime}=\left\{\tau \in \mathscr{S}: 1 \times f_{j_{1}} \in \tau\right\} \text {. }
$$

Proposition $5.16 \mathscr{S}^{\prime}$ is not empty.

Proof Suppose $\tau \in \mathscr{S}$. Let $P^{\prime}$ be the path in $T(\tau)$ from 3 to 4. By Proposition 5.15 (a) and the proof of Proposition 5.11, $P^{\prime}$ has one of the following forms.

(i') $P^{\prime}=\left\{3 \times f_{j_{1}^{\prime}}, 4 \times f_{j_{1}^{\prime}}\right\}, j_{1}^{\prime} \neq 1,2$.

(ii') $P^{\prime}=\left\{3 \times f_{j_{1}^{\prime}}, 2 \times f_{j_{1}^{\prime}}, 2 \times f_{j_{2}^{\prime}}, 4 \times f_{j_{2}^{\prime}}\right\}, j_{1}^{\prime} \neq 1, j_{2}^{\prime} \neq 2$.

(iii') $P^{\prime}=\left\{3 \times f_{j_{1}^{\prime}}, 1 \times f_{j_{1}^{\prime}}, 1 \times f_{j_{2}^{\prime}}, 4 \times f_{j_{2}}\right\}, j_{1}^{\prime} \neq 1, j_{2}^{\prime} \neq 2$.

Suppose we have (iii'). Then by the proof of Proposition 5.11, there is some $\tau^{\prime} \in$ $\mathscr{T}\left(X^{-}\right)_{1}$ with $P^{\prime} \subseteq \tau^{\prime}$. Since $P^{\prime} \neq P$, we have $\sigma_{0} \nsubseteq \tau^{\prime}$. This contradicts $\tau_{0}$-goodness. Hence $\left(\mathrm{i}^{\prime}\right)$ or $\left(\mathrm{ii}^{\prime \prime}\right)$ must hold.

Now, let $\tau^{\prime}$ be the minimal element of $\mathscr{T}\left(X^{-} \cup Y^{-} \cup P^{\prime}\right)$ with respect to $\preceq_{4}$. Since $P^{\prime} \subseteq \tau^{\prime}$, we have $\tau^{\prime} \in \mathscr{S}$. By Proposition 4.11, we have $1 \times f_{j} \in \tau^{\prime}$ for $j=1,2, j_{1}$, or $j_{r}^{\prime}$ for some $r{ }^{7}$ By Proposition 5.15 (c), we have $j \neq 1,2$.

Suppose $j=j_{r}^{\prime}$. First assume $\left(\mathrm{i}^{\prime}\right)$ is true. Then $1 \times f_{j_{1}^{\prime}} \in \tau^{\prime}$. On the other hand, we also have $2 \times f_{j^{\prime}} \in \tau^{\prime}$ for some $j=1,2, j_{r}$ or $j_{1}^{\prime}$, and so by Propositions 5.15 (b) and (c), we have $2 \times f_{j_{1}^{\prime}} \in \tau^{\prime}$. This contradicts $\tau_{\mathrm{I}}$-goodness(b). If (ii') is true, then we immediately have a contradiction to $\tau_{\mathrm{I}}$-goodness(b). Hence $j=j_{1}$. So $\tau^{\prime} \in \mathscr{S}^{\prime}$, as desired.

Note that in this proof, we showed that $1 \times f_{j_{r}^{\prime}} \notin \tau^{\prime}$ for any $r$. Since $1 \times f_{j_{1}} \in \tau^{\prime}$, and in Case (ii), $1 \times f_{j_{2}} \in \tau^{\prime}$ since $Y^{-} \subseteq \tau^{\prime}$, it follows that $f_{j_{r}^{\prime}} \neq j_{1}, j_{2}$ for all $r$.

\footnotetext{
7 This is true in both Cases (i) and (ii). In Case (ii), consider the 2-alternating path from 4 to 1 . The second to last edge of this path must be $i \times f_{j} \in X^{-} \cup Y^{-} \cup P^{\prime}$ for some $i \neq 1$; therefore $j=1,2, j_{1}$, or $j_{r}^{\prime}$.
} 
Now, choose $\tau$ to be a maximal element of $\mathscr{S}^{\prime}$ with respect to $\preceq_{23}$. Let $P^{\prime}$ be the path in $T(\tau)$ from 3 to 4 . Then as in the previous proof, either (i') or (ii') is true. We consider these cases separately.

Subcase 3.1: $P^{\prime}$ has form (i'). As noted in the previous proof, we have $j_{1}^{\prime} \neq j_{1}, j_{2}$. In Case (i), let $Z=\left\{Z^{+}, Z^{-}\right\}$be the circuit

$$
\begin{aligned}
& Z^{-}=\left\{3 \times f_{j_{1}^{\prime}}, 4 \times f_{j_{1}}\right\}, \\
& Z^{+}=\left\{4 \times f_{j_{1}^{\prime}}, 3 \times f_{j_{1}}\right\},
\end{aligned}
$$

and in Case (ii), let $Z$ be the circuit

$$
\begin{aligned}
& Z^{-}=\left\{3 \times f_{j_{1}^{\prime}}, 4 \times f_{j_{1}}, 1 \times f_{j_{2}}\right\}, \\
& Z^{+}=\left\{4 \times f_{j_{1}^{\prime}}, 1 \times f_{j_{1}}, 3 \times f_{j_{2}}\right\} .
\end{aligned}
$$

Let $\pi=Z \backslash\left\{3 \times f_{j_{t}}\right\}$, where $t=1$ in Case (i) and $t=2$ in Case (ii). Then $\pi$ is a maximal simplex of $\mathscr{T}_{Z}^{+}$. Since $1 \times f_{j_{1}} \in \tau$ by the definition of $\mathscr{S}^{\prime}$, we have $\pi \cup X^{-} \cup\left\{1 \times f_{j_{1}}\right\}=X^{-} \cup Y^{-} \cup P^{\prime} \cup\left\{1 \times f_{j_{1}}\right\} \subseteq \tau$. Let $\tau_{*}$ be the minimal element of $\mathscr{T}\left(X^{-} \cup Y^{-} \cup P^{\prime} \cup\left\{1 \times f_{j_{1}}\right\}\right)$ with respect to $\preceq_{3}$. By the proof of Proposition 5.16, we have $2 \times f_{j_{1}^{\prime}} \in \tau_{*}$. So by Proposition 4.11, $\tau_{*}$ is minimal in $\mathscr{T}\left(Z^{-}\right)$with respect to $\preceq 3$.

Since $P^{\prime} \cup\left\{1 \times f_{j_{1}}, 4 \times f_{j_{1}}\right\} \subseteq \tau, \tau^{*}$, by Lemma 4.7 we have $\tau \sim_{23} \tau_{*}$. Hence $\tau_{*}$ is maximal in $\mathscr{S}^{\prime}$ with respect to $\preceq_{23}$. We can thus redefine $\tau$ as $\tau=\tau^{*}$.

We claim that $\mathscr{T}\left(Z^{-}\right) \subseteq \mathscr{T}\left(Y^{-}\right)$. Suppose $\tau^{\prime} \in \mathscr{T}\left(Z^{-}\right)$. We have $Y^{-} \backslash\{3 \times$ $\left.f_{1}\right\} \subseteq Z^{-} \subseteq \tau^{\prime}$. Also, since $\tau$ is minimal in $\mathscr{T}\left(Z^{-}\right)$with respect to $\preceq_{3}$, we have $N_{\tau}(3) \subseteq N_{\tau^{\prime}}(3)$. Hence $3 \times f_{1} \in \tau^{\prime}$. So $\tau^{\prime} \in \mathscr{T}\left(Y^{-}\right)$.

We next claim that $\pi^{\prime}:=Z \backslash\left\{4 \times f_{j_{1}^{\prime}}\right\} \in \mathscr{T}$. By Proposition 3.3, there exists a maximal simplex $\tau^{\prime} \in \mathscr{T}\left(Z^{-}\right)$with $\tau^{\prime}=\tau \backslash\left\{4 \times f_{j_{1}^{\prime}}\right\} \cup\left\{i \times f_{j}\right\}$ for some $i \times f_{j}$ where $i \in\{2,3\}$. Then $\tau \prec_{3} \tau^{\prime}$, so by the definition of $\tau$, we must have $\tau^{\prime} \notin \mathscr{S}^{\prime}$. Hence, we must have $i \times f_{j}=3 \times f_{j_{t}}$. Thus $\pi^{\prime} \subseteq \tau^{\prime}$, as desired.

We finally claim that $\mathscr{T}$ has a flip supported on $\left(Z^{+}, Z^{-}\right)$. Suppose the contrary. Since $\pi^{\prime} \in \mathscr{T}$, by Proposition 4.12, there is some $\tau^{\prime} \in \mathscr{T}\left(Z^{-}\right)$with $\left|\tau^{\prime} \cap Z^{+}\right| \leq$ $\left|Z^{+}\right|-2$ and $4 \times f_{j_{1}^{\prime}} \notin \tau^{\prime}$. As shown above, $\tau^{\prime} \in \mathscr{T}\left(Y^{-}\right)$. In addition, since $\tau$ is minimal in $\mathscr{T}\left(Z^{-}\right)$with respect to $\preceq_{3}$, we have $N_{\tau}\left(f_{1}\right) \supseteq N_{\tau^{\prime}}\left(f_{1}\right)$, so $4 \times f_{1} \notin \tau^{\prime}$. It follows that $\left|\tau^{\prime} \cap Y^{+}\right| \leq\left|\tau^{\prime} \cap Z^{+}\right| \leq\left|Z^{+}\right|-2=\left|Y^{+}\right|-2$. Hence, $\tau^{\prime} \in \mathscr{S}$.

Let $P^{\prime \prime}$ be the path from 3 to 4 in $T\left(\tau^{\prime}\right)$, and let $\tau^{\prime \prime}$ be the minimal element of $\mathscr{T}\left(X^{-} \cup Y^{-} \cup P^{\prime \prime}\right)$ with respect to $\preceq_{4}$. By the proof of Proposition $5.16, \tau^{\prime \prime} \in \mathscr{S}^{\prime}$. Since $4 \times f_{j_{1}^{\prime}} \notin P^{\prime \prime}$ by the definition of $\tau^{\prime}$, we have $P^{\prime} \nsubseteq \tau^{\prime \prime}$. Thus, by Lemma 4.7, we have $\tau \nsim_{23} \tau^{\prime \prime}$. It follows that $\tau \prec_{23} \tau^{\prime \prime}$, which contradicts the maximality of $\tau$ in $\mathscr{S}^{\prime}$ with respect to $\preceq_{23}$.

Hence, $\mathscr{T}$ has a flip supported on $\left(Z^{+}, Z^{-}\right)$. Let $\mathscr{T}^{\prime}$ be the result of this flip. In both cases, we have

$$
\mathscr{T}^{\prime}\left(Y^{-}\right)^{*}=\mathscr{T}\left(Y^{-}\right)^{*} \backslash \mathscr{T}\left(Z^{-}\right)^{*} \cup \Psi\left(\mathscr{T}\left(Z \backslash\left\{4 \times f_{j_{1}^{\prime}}\right\}\right)^{*}\right) .
$$


Hence $\left|\mathscr{T}^{\prime}\left(Y^{-}\right)^{*}\right|<\left|\mathscr{T}\left(Y^{-}\right)^{*}\right|$. It is also clear from $\Psi$ that $\left|\mathscr{T}^{\prime}\left(X^{-}\right)^{*}\right|=\left|\mathscr{T}\left(X^{-}\right)^{*}\right|$. By Proposition 5.5 (a) in Case (i) and Proposition 5.4 (a) in Case (ii), $\left|\left(\mathscr{T}^{\prime}\right)^{\mathrm{I}}\right| \leq\left|\mathscr{T}^{\mathrm{I}}\right|$. It is easy to check that if $\Psi(\tau) \in \mathscr{T}^{\prime \prime}\left(X^{-}\right)_{2}$, then $\tau \in \mathscr{T}^{\prime}\left(X^{-}\right)_{2}$. It is also easy to check $\tau_{\mathrm{I}}$-goodness and $\tau_{0}$-goodness.

Finally, to see that $\tau_{\mathrm{I}}, \tau_{0} \in \mathscr{T}^{\prime}$, it suffices to show $\tau_{\mathrm{I}}, \tau_{0} \notin \mathscr{T}\left(Z^{-}\right)$. Since $\tau$ is minimal in $\mathscr{T}\left(Z^{-}\right)$with respect to $\preceq_{3}$, for any $\tau^{\prime} \in \mathscr{T}\left(Z^{-}\right)$we have $N_{\tau}\left(f_{1}\right) \supseteq$ $N_{\tau^{\prime}}\left(f_{1}\right)$ and hence $2 \times f_{1} \notin \tau^{\prime}$. Thus $\tau_{\mathrm{I}}, \tau_{0} \notin \mathscr{T}\left(Z^{-}\right)$.

Subcase 3.2: $P^{\prime}$ has form (ii'). In Case (i), let $Z=\left\{Z^{+}, Z^{-}\right\}$be the circuit

$$
\begin{aligned}
& Z^{-}=\left\{3 \times f_{j_{1}^{\prime}}, 2 \times f_{j_{2}^{\prime}}, 4 \times f_{j_{1}}\right\}, \\
& Z^{+}=\left\{2 \times f_{j_{1}^{\prime}}, 4 \times f_{j_{2}^{\prime}}, 3 \times f_{j_{1}}\right\},
\end{aligned}
$$

and in Case (ii),

$$
\begin{aligned}
& Z^{-}=\left\{3 \times f_{j_{1}^{\prime}}, 2 \times f_{j_{2}^{\prime}}, 4 \times f_{j_{1}}, 1 \times f_{j_{2}}\right\} \\
& Z^{+}=\left\{2 \times f_{j_{1}^{\prime}}, 4 \times f_{j_{2}^{\prime}}, 1 \times f_{j_{1}}, 3 \times f_{j_{2}}\right\}
\end{aligned}
$$

Let $\pi=Z \backslash\left\{3 \times f_{j_{t}}\right\}$, where $t=1$ in Case (i) and $t=2$ in Case (ii). Then $\pi$ is a maximal simplex of $\mathscr{T}_{Z}^{+}$and $\pi \cup X^{-} \cup\left\{1 \times f_{j_{1}}\right\} \subseteq \tau$. By Proposition 4.11, $\pi$ is minimal in $\mathscr{T}\left(X^{-}\right)$with respect to $\preceq_{3}$.

By the same arguments as in the previous subcase, we have $\mathscr{T}\left(Z^{-}\right) \subseteq \mathscr{T}\left(Y^{-}\right)$, $Z \backslash\left\{4 \times f_{j_{2}^{\prime}}\right\} \in \mathscr{T}$, and $\mathscr{T}$ has a flip supported on $\left(Z^{+}, Z^{-}\right)$. The remainder of Claim 5.14 also follows the arguments of the previous subcase. This concludes the proof of Phase I.

\subsection{Phase II}

Let $\mathscr{T}$ be a triangulation of $\Delta^{3} \times \Delta^{n-1}$. Let $\mathscr{T}^{\mathrm{II}}$ be the set of all maximal simplices $\tau \in \mathscr{T}^{*}$ for which there is some $f_{j} \in \Delta^{n-1}$ with $\{1,2\} \subseteq N_{\tau}\left(f_{j}\right)$ and $\{3,4\} \nsubseteq$ $N_{\tau}\left(f_{j}\right)$.

Claim 5.17 $\mathscr{T}$ is flip-connected to a triangulation $\mathscr{T}^{\prime}$ with $\left(\mathscr{T}^{\prime}\right)^{\mathrm{II}}=\varnothing$.

The proof uses similar arguments to Phase I but is much easier. The reader can find it in [8].

\subsection{Phase III}

To complete the proof, we now define a specific triangulation $\mathscr{T}_{0}$ and show that all triangulations of $\Delta^{3} \times \Delta^{n-1}$ are flip-connected to it.

Let $\leq_{0}$ be the total ordering on $\Delta^{n-1}$ such that $f_{i}<_{0} f_{j}$ if $i<j$. We have the following.

Proposition 5.18 There is a unique triangulation $\mathscr{T}_{0}$ of $\Delta^{3} \times \Delta^{n-1}$ such that $\left(\mathscr{T}_{0}\right)^{\mathrm{II}}=$ $\emptyset$ and the orders $\leq \mathscr{T}_{0[12]}$ and $\leq \mathscr{T}_{0[34]}$ are both the same as $\leq_{0}$. 
Claim 5.19 If $\mathscr{T}$ is a triangulation of $\Delta^{3} \times \Delta^{n-1}$, then $\mathscr{T}$ is flip-connected to $\mathscr{T}_{0}$.

The proofs are found in [8]. This concludes the proof of the theorem.

Acknowledgements Open access funding provided by Max Planck Society. The author would like to thank Francisco Santos for his valuable feedback and his insight in Remark 4.2. The author would also like to thank Alexander Postnikov for introducing this problem to him and for helpful discussions. Finally, the author would like to thank the anonymous reviewers for their careful reading and useful suggestions. This material is based upon work supported by the National Science Foundation Graduate Research Fellowship under Grant No. 1122374.

Open Access This article is distributed under the terms of the Creative Commons Attribution 4.0 International License (http://creativecommons.org/licenses/by/4.0/), which permits unrestricted use, distribution, and reproduction in any medium, provided you give appropriate credit to the original author(s) and the source, provide a link to the Creative Commons license, and indicate if changes were made.

\section{References}

1. Ardila, F., Billey, S.: Flag arrangements and triangulations of products of simplices. Adv. Math. 214(2), 495-524 (2007)

2. Ardila, F., Ceballos, C.: Acyclic systems of permutations and fine mixed subdivisions of simplices. Discrete Comput. Geom. 49(3), 485-510 (2013)

3. Ardila, F., Develin, M.: Tropical hyperplane arrangements and oriented matroids. Math. Z. 262(4), 795-816 (2009)

4. De Loera, J.A., Rambau, J., Santos, F.: Triangulations: Structures for Algorithms and Applications. Algorithms and Computation in Mathematics, vol. 25. Springer, Berlin (2010)

5. Develin, M., Sturmfels, B.: Tropical convexity. Doc. Math. 9, 1-27 (2004)

6. Gel'fand, I.M., Kapranov, M.M., Zelevinsky, A.V.: Discriminants, Resultants and Multidimensional Determinants. Mathematics: Theory \& Applications. Birkhäuser, Boston (1994)

7. Horn, S.: A topological representation theorem for tropical oriented matroids. In: Proceedings of the 24th International Conference on Formal Power Series and Algebraic Combinatorics (FPSAC 2012). Discrete Mathematics \& Theoretical Computer Science Proceedings, AR, pp. 135-146. DMTCS, Nancy (2012)

8. Liu, G.: Flip-connectivity of triangulations of the product of a tetrahedron and simplex (2016). arXiv: 1601.06031

9. Liu, G.: A zonotope and a product of two simplices with disconnected flip graphs. Discrete Comput. Geom. 59(4), 810-842 (2018)

10. Maclagan, D., Thomas, R.R.: Combinatorics of the toric Hilbert scheme. Discrete Comput. Geom. 27(2), 249-272 (2002)

11. Oh, S., Yoo, H.: Triangulations of $\Delta_{n-1} \times \Delta_{d-1}$ and tropical oriented matroids. In: Proceedings of the 23th International Conference on Formal Power Series and Algebraic Combinatorics (FPSAC 2011). Discrete Mathematics \& Theoretical Computer Science Proceedings, AR, pp. 717-728. DMTCS, Nancy (2011)

12. Santos, F.: A point set whose space of triangulations is disconnected. J. Am. Math. Soc. 13(3), 611-637 (2000)

13. Santos, F.: Triangulations of Oriented Matroids. Memoirs of the American Mathematical Society, vol. 156(741). American Mathematical Society, Providence (2002)

14. Santos, F.: The Cayley trick and triangulations of products of simplices. In: Barvinok, A., et al. (eds.) Integer Points in Polyhedra-Geometry, Number Theory, Algebra, Optimization. Contemporary Mathematics, vol. 374, pp. 151-177. American Mathematical Society, Providence (2005)

15. Sturmfels, B.: Gröbner Bases and Convex Polytopes. University Lecture Series, vol. 8. American Mathematical Society, Providence (1996)

Publisher's Note Springer Nature remains neutral with regard to jurisdictional claims in published maps and institutional affiliations. 